\title{
Reactions of Amidinate-supported Silylene with Organoboron Dihalides
}

Jiancheng Li, ${ }^{\ddagger}$ Yashuai Liu, ${ }^{\ddagger}$ Subrata Kundu, ${ }^{\ddagger}{ }^{\ddagger}$ Helena Keil, ${ }^{\ddagger}$ Hongping Zhu, ${ }^{\star}+$ Regine Herbst-Irmer, ${ }^{\ddagger}$ Dietmar Stalke, ${ }^{*}$ Herbert W. Roesky, ${ }^{*}$

† State Key Laboratory of Physical Chemistry of Solid Surfaces, National Engineering Laboratory for Green Chemical Productions of Alcohols-Ethers-Esters, College of Chemistry and Chemical Engineering, Xiamen University, Xiamen 361005, China

‡ Institut für Anorganische Chemie, Georg-August-Universität Göttingen, Tammannstr. 4, 37077 Göttingen, Germany

$\S$ Department of Chemistry, Indian Institute of Technology Delhi, Hauz Khas, New Delhi, 110016, India.

\section{Contents:}

1. Experimental Section

2. The Proposed Reaction Mechanism.

3. X-Ray Crystallographic Analysis of Complexes 1, 2, 3 and 7

4. References 


\section{Experimental Section}

All manipulations were carried out under a dry argon or nitrogen atmosphere using Schlenk line and glovebox techniques. Solvents including THF, diethyl ether, toluene and $n$-hexane were dried by refluxing with sodium/potassium benzophenone under $\mathrm{N}_{2}$ prior to use. The NMR $\left({ }^{1} \mathrm{H},{ }^{13} \mathrm{C},{ }^{11} \mathrm{~B},{ }^{29} \mathrm{Si}\right)$ spectra were recorded on Bruker Avance $300 \mathrm{MHz}$ and/or Bruker Avance $500 \mathrm{MHz}$ spectrometer. Melting points of compounds were measured in a sealed glass tube using the Büchi- 540 instrument. Elemental analysis were performed by the Analytisches Labor des Instituts für Anorganische Chemie der Universität Göttingen. Commercial reagents were purchased from Aldrich, Acros or Alfa-Aesar Chemical Co. and used as received. $\mathrm{LSiCl}^{1}, \mathrm{LSiMes}^{2},\left(\mathrm{Me}_{3} \mathrm{Si}_{2}\right)_{2} \mathrm{NBCl}_{2}{ }^{3}, \mathrm{MesBCl}_{2}{ }^{4}$ and $\mathrm{MesBBr}_{2}{ }^{4}(\mathrm{~L}=$ $\left.\mathrm{PhC}(\mathrm{NtBu})_{2}\right)$, Mes $=2,4,6-\mathrm{Me}_{3} \mathrm{C}_{6} \mathrm{H}_{2}$ ) were prepared according to the literature.

$\mathrm{LClSi} \rightarrow \mathrm{BPhCl}_{2}$ (1): To a solution of $\mathrm{LSiCl}(885 \mathrm{mg}, 3 \mathrm{mmol})$ in toluene $(40 \mathrm{~mL})$ at $-78{ }^{\circ} \mathrm{C}$, a solution of $\mathrm{PhBCl}_{2}(0.38 \mathrm{~mL}, 3 \mathrm{mmol})$ in toluene was added dropwise under stirring. The mixture was slowly warmed to room temperature and stirred overnight. After filtration, the colorless solution was concentrated to afford an off-white solid, which was collected and washed with $n$-hexane (5 mL). Yield: 1.17 g (86\%). Mp: $98{ }^{\circ} \mathrm{C} .{ }^{1} \mathrm{H}$ NMR (THF-D $\left., 298 \mathrm{~K}, 300 \mathrm{MHz}, \mathrm{ppm}\right): \delta=7.86-7.13(\mathrm{~m}, 10 \mathrm{H}, \mathrm{Ph}-\mathrm{H}), 1.14(\mathrm{~s}, 18 \mathrm{H}, \mathrm{CMe}) .{ }^{13} \mathrm{C}\left\{{ }^{1} \mathrm{H}\right\} \mathrm{NMR}$ $\left(\mathrm{THF}-\mathrm{D}_{8}, 298 \mathrm{~K}, 75 \mathrm{MHz}, \mathrm{ppm}\right): \delta=179.5\left(\mathrm{PhC}(\mathrm{NtBu})_{2}\right), 132.9,131.7,128.6,128.4,128.3,128.2,126.7,126.2(\mathrm{Ph}-\mathrm{C}), 55.8(\mathrm{NCMe})$, $30.2\left(\mathrm{NCMe}_{3}\right) .{ }^{29} \mathrm{Si} \mathrm{NMR}\left(\mathrm{THF}-\mathrm{D}_{8}, 298 \mathrm{~K}, 99 \mathrm{MHz}, \mathrm{ppm}\right): \delta=4.1$ (br, SiCl $\left.)_{3}\right){ }^{11} \mathrm{~B}\left\{{ }^{1} \mathrm{H}\right\} \mathrm{NMR}\left(\mathrm{THF}-\mathrm{D}_{8}, 298 \mathrm{~K}, 160.5 \mathrm{MHz}, \mathrm{ppm}\right): \delta=-0.03$ $\left(\mathrm{PhBCl}_{2}\right)$. Anal. Calcd (\%) for $\mathrm{C}_{21} \mathrm{H}_{28} \mathrm{BCl}_{3} \mathrm{~N}_{2} \mathrm{Si}\left(\mathrm{M}_{\mathrm{r}}=452.12\right)$ : C, 55.59; H, 6.22; N, 6.17; Found: $\mathrm{C}, 55.34 ; \mathrm{H}, 6.29 ; \mathrm{N}, 6.41$. X-ray quality single-crystals were grown from the solution in $n$-hexane at $-26^{\circ} \mathrm{C}$ for one day.

$\mathrm{LB}(\mathrm{Ph}) \mathrm{SiCl}_{3}(2)$ : The solution of $1(452 \mathrm{mg}, 1 \mathrm{mmol})$ in toluene $(40 \mathrm{~mL})$ was heated to $65^{\circ} \mathrm{C}$ and stirred overnight at this temperature. After filtration, the colorless solution was concentrated to ca. $3 \mathrm{~mL}$. The solution was cooled to $-20^{\circ} \mathrm{C}$ for one day to afford colorless crystals which were suitable for X-ray diffraction study. Yield: $0.42 \mathrm{~g}(91 \%)$. Mp: $94{ }^{\circ} \mathrm{C} .{ }^{1} \mathrm{H} \mathrm{NMR}\left(\mathrm{C}_{6} \mathrm{D}_{6}, 298 \mathrm{~K}, 400 \mathrm{MHz}, \mathrm{ppm}\right): \delta=$ 7.97-6.94 (m, $10 \mathrm{H}, \mathrm{Ph}-\mathrm{H}), 1.02\left(\mathrm{~s}, 18 \mathrm{H}, \mathrm{CMe}_{3}\right) .{ }^{13} \mathrm{C}\left\{{ }^{1} \mathrm{H}\right\} \mathrm{NMR}\left(\mathrm{C}_{6} \mathrm{D}_{6}, 298 \mathrm{~K}, 100 \mathrm{MHz}, \mathrm{ppm}\right): \delta=168.6\left(\mathrm{PhC}(\mathrm{NtBu})_{2}\right), 133.5,131.5$, 130.0, 129.1, 128.6, 128.2, $127.9(\mathrm{Ph}-\mathrm{C}), 54.1\left(\mathrm{NCMe}_{3}\right), 31.1\left(\mathrm{NCMe}_{3}\right) .{ }^{29} \mathrm{Si} \mathrm{NMR}\left(\mathrm{C}_{6} \mathrm{D}_{6}, 298 \mathrm{~K}, 99 \mathrm{MHz}, \mathrm{ppm}\right): \delta=28.7(\mathrm{br}, \mathrm{SiCl} 3) .{ }^{11} \mathrm{~B}$ $\left\{{ }^{1} \mathrm{H}\right\} \operatorname{NMR}\left(\mathrm{C}_{6} \mathrm{D}_{6}, 298 \mathrm{~K}, 128 \mathrm{MHz}, \mathrm{ppm}\right): \delta=3.5\left(\mathrm{BSiCl}_{3}\right)$. Anal. Calcd (\%) for $\mathrm{C}_{21} \mathrm{H}_{28} \mathrm{BCl}_{3} \mathrm{~N}_{2} \mathrm{Si}\left(\mathrm{Mr}_{\mathrm{r}}=452.12\right): \mathrm{C}, 55.59 ; \mathrm{H}, 6.22 ; \mathrm{N}, 6.17$; Found: C, 55.52; H, 6.36; N, 6.03 .

$\mathrm{LB}(\mathrm{Mes}) \mathrm{SiCl}_{3}$ (3): To a solution of $\mathrm{LSiCl}(1.475 \mathrm{~g}, 5 \mathrm{mmol})$ in toluene $(40 \mathrm{~mL})$ at $-78{ }^{\circ} \mathrm{C}$, a solution of $\mathrm{MesBCl}_{2}(1.00 \mathrm{~g}, 5 \mathrm{mmol})$ in toluene $(20 \mathrm{~mL})$ was added dropwise under stirring. The mixture was slowly warmed to room temperature and stirred overnight. After filtration, the colorless solution was concentrated to ca. $5 \mathrm{~mL}$. The solution was cooled to $-26{ }^{\circ} \mathrm{C}$ for $12 \mathrm{~h}$ to afford colorless crystals which were suitable for X-ray diffraction study. Yield: $2.27 \mathrm{~g}(92 \%)$. Mp: $181{ }^{\circ} \mathrm{C}$. ${ }^{1} \mathrm{H}$ NMR (THF-D $\left., 298 \mathrm{~K}, 300 \mathrm{MHz}, \mathrm{ppm}\right): \delta=8.1-7.5$ (m, $5 \mathrm{H}, \mathrm{Ph}-\mathrm{H}), 6.83\left(\mathrm{~s}, 1 \mathrm{H}, \mathrm{C}_{6} \mathrm{H}_{2}\right.$ ), 6.80 (s, $1 \mathrm{H}, \mathrm{C}_{6} \mathrm{H}_{2}$ ), 2.93 (s, $\left.3 \mathrm{H}, \mathrm{Ar}-\mathrm{Me}\right), 2.74$ (s, $\left.3 \mathrm{H}, \mathrm{Ar}-\mathrm{Me}\right), 2.24$ (s, $\left.3 \mathrm{H}, \mathrm{Ar}-\mathrm{Me}\right), 1.19$ (s, $36 \mathrm{H}$, $\left.\mathrm{CMe}_{3}\right) .{ }^{13} \mathrm{C}\left\{{ }^{1} \mathrm{H}\right\}$ NMR $\left(\mathrm{THF}_{-} \mathrm{D}_{8}, 298 \mathrm{~K}, 75 \mathrm{MHz}, \mathrm{ppm}\right): \delta=166.4\left(\mathrm{PhC}(\mathrm{NtBu})_{2}\right), 143.6,142.5,136.5,131.2,130.7,129.9,129.5,128.2$ (Ph-C), 54.5 ( $\mathrm{NCMe}_{3}$ ), 30.2 (NCMe $), 27.1$ (Ar-Me), 22.0 (Ar-Me), 19.8 (Ar-Me). ${ }^{29} \mathrm{Si} \mathrm{NMR}\left(\mathrm{THF}-\mathrm{D}_{8}, 298 \mathrm{~K}, 99 \mathrm{MHz}, \mathrm{ppm}\right): \delta=24.5$ (br, $\left.\mathrm{SiCl}_{3}\right) .{ }^{11} \mathrm{~B}\left\{{ }^{1} \mathrm{H}\right\} \mathrm{NMR}\left(\mathrm{THF}-\mathrm{D}_{8}, 298 \mathrm{~K}, 160.5 \mathrm{MHz}, \mathrm{ppm}\right): \delta=3.0\left(\mathrm{BSiCl}_{3}\right)$. Anal. Calcd $(\%)$ for $\mathrm{C}_{24} \mathrm{H}_{34} \mathrm{BCl}_{3} \mathrm{~N}_{2} \mathrm{Si}\left(M_{\mathrm{r}}=494.16\right): \mathrm{C}, 58.14$; $H, 6.91 ; \mathrm{N}, 5.65$; Found: C, 57.86; H, 7.12; N, 5.82 .

$\mathrm{LB}\left[\mathrm{N}\left(\mathrm{SiMe}_{3}\right)_{2}\right] \mathrm{SiCl}_{3}(4)$ : To a solution of $\mathrm{LSiCl}(148 \mathrm{mg}, 0.5 \mathrm{mmol})$ in $\mathrm{C}_{6} \mathrm{D}_{6}(0.5 \mathrm{~mL})$ in an NMR-tube at room temperature, a solution of $\left(\mathrm{Me}_{3} \mathrm{Si}_{2}\right)_{\mathrm{NBCl}_{2}}(120 \mathrm{mg}, 0.5 \mathrm{mmol})$ in $\mathrm{C}_{6} \mathrm{D}_{6}(0.2 \mathrm{~mL})$ was added. The mixture was shaken for 15 minutes. After filtration, the filtrate was concentrated to afford an off-white solid, which was washed with pre-cold $n$-hexane $(2 \mathrm{~mL})$. Yield: $0.22 \mathrm{~g}(83 \%)$. Mp: $86{ }^{\circ} \mathrm{C}$ (dec.). ${ }^{1} \mathrm{H}$ $\operatorname{NMR}\left(\mathrm{C}_{6} \mathrm{D}_{6}, 298 \mathrm{~K}, 300 \mathrm{MHz}, \mathrm{ppm}\right): \delta=8.00-6.90(\mathrm{~m}, 10 \mathrm{H}, \mathrm{Ph}-\mathrm{H}), 1.27$ (s, 36 H, CMe $), 0.61$ (s, $\left.9 \mathrm{H}, \mathrm{SiMe}\right), 0.49(\mathrm{~s}, 9 \mathrm{H}, \mathrm{SiMe}) .{ }^{13} \mathrm{C}$ $\left\{{ }^{1} \mathrm{H}\right\} \operatorname{NMR}\left(\mathrm{C}_{6} \mathrm{D}_{6}, 298 \mathrm{~K}, 75 \mathrm{MHz}, \mathrm{ppm}\right): \delta=167.2\left(\mathrm{PhC}(\mathrm{N} t \mathrm{Bu})_{2}\right), 141.6,131.1,130.1,129.0,128.4(\mathrm{Ph}-\mathrm{C}), 54.5\left(\mathrm{NCMe}_{3}\right), 31.1$ $\left(\mathrm{NCMe}_{3}\right), 7.1\left(\mathrm{SiMe}_{3}\right), 6.8\left(\mathrm{SiMe}_{3}\right) .{ }^{29} \mathrm{Si} \mathrm{NMR}\left(\mathrm{C}_{6} \mathrm{D}_{6}, 298 \mathrm{~K}, 99 \mathrm{MHz}, \mathrm{ppm}\right): \delta=21.3\left(\mathrm{~m}, \mathrm{BSiCl}_{3}\right), 3.94\left(\mathrm{NSiMe}_{3}\right), 3.54\left(\mathrm{NSiMe}_{3}\right) .{ }^{11} \mathrm{~B}\left\{{ }^{1} \mathrm{H}\right\}$ $\operatorname{NMR}\left(\mathrm{C}_{6} \mathrm{D}_{6}, 298 \mathrm{~K}, 96 \mathrm{MHz}, \mathrm{ppm}\right): \delta=4.5\left(\mathrm{br}, \mathrm{BSiCl}_{3}\right)$. Anal. Calcd $(\%)$ for $\mathrm{C}_{21} \mathrm{H}_{41} \mathrm{BCl}_{3} \mathrm{~N}_{3} \mathrm{Si}_{3}\left(M_{\mathrm{r}}=537.00\right): \mathrm{C}, 46.97 ; \mathrm{H}, 7.70 ; \mathrm{N}, 7.83$; Found: C, 46.38; H, 7.15; N, 7.64 .

$\mathrm{LB}(\mathrm{Ph}) \mathrm{SiCl}_{2} \mathrm{Mes}(5)$ : To a solution of LSiMes $(378 \mathrm{mg}, 1 \mathrm{mmol})$ in toluene $(40 \mathrm{~mL})$ at $-78^{\circ} \mathrm{C}$, a solution of $\mathrm{PhBCl}_{2}(159 \mathrm{mg}, 1 \mathrm{mmol})$ in toluene $(20 \mathrm{~mL})$ was added dropwise under stirring. The mixture was slowly warmed to room temperature and stirred overnight. After filtration, the colorless solution was concentrated to afford an off-white solid, which was washed with $n$-hexane $(2 \mathrm{~mL})$. Yield: $0.47 \mathrm{~g}$ (87\%). Mp: $189^{\circ} \mathrm{C} .{ }^{1} \mathrm{H}$ NMR $\left(\mathrm{C}_{6} \mathrm{D}_{6}, 298 \mathrm{~K}, 400 \mathrm{MHz}, \mathrm{ppm}\right): \delta=8.24-8.23(\mathrm{~m}, 2 \mathrm{H}, \mathrm{Ph}-H), 7.64-7.60(\mathrm{~m}, 1 \mathrm{H}, \mathrm{Ph}-\mathrm{H}), 7.42-7.36(\mathrm{~m}, 2 \mathrm{H}$, $\mathrm{Ph}-H), 7.23-7.28(\mathrm{~m}, 1 \mathrm{H}, \mathrm{Ph}-H), 7.03-6.86(\mathrm{~m}, 4 \mathrm{H}, \mathrm{Ph}-H), 6.67$ (s, $\left.2 \mathrm{H}, \mathrm{C}_{6} \mathrm{H}_{2}\right), 2.84(\mathrm{~s}, 6 \mathrm{H}, \mathrm{Ar}-\mathrm{Me}), 1.99(\mathrm{~s}, 3 \mathrm{H}, \mathrm{Ar}-\mathrm{Me}), 1.00(\mathrm{~s}, 18 \mathrm{H}$, $\left.\mathrm{CMe}_{3}\right) .{ }^{13} \mathrm{C}\left\{{ }^{1} \mathrm{H}\right\} \mathrm{NMR}\left(\mathrm{C}_{6} \mathrm{D}_{6}, 298 \mathrm{~K}, 100 \mathrm{MHz}, \mathrm{ppm}\right): \delta=166.9\left(\mathrm{PhC}(\mathrm{NtBu})_{2}\right), 144.4,139.7,134.0,133.2,132.4,130.2,129.7,129.1$, 128.9, 127.0 (Ph-C and Ar-C), $53.6\left(\mathrm{NCMe}_{3}\right), 30.8\left(\mathrm{NCMe}_{3}\right), 26.5(\mathrm{Ar}-\mathrm{Me}), 20.5(\mathrm{Ar}-\mathrm{Me}) .{ }^{29} \mathrm{Si} \mathrm{NMR}\left(\mathrm{C}_{6} \mathrm{D}_{6}, 298 \mathrm{~K}, 99 \mathrm{MHz}, \mathrm{ppm}\right): \delta=$ 28.0 (br, SiCl $\mathrm{Mes}) .{ }^{11} \mathrm{~B}\left\{{ }^{1} \mathrm{H}\right\}$ NMR $\left(\mathrm{C}_{6} \mathrm{D}_{6}, 298 \mathrm{~K}, 128 \mathrm{MHz}, \mathrm{ppm}\right)$ : $\delta=5.3\left(\mathrm{br}, B S i C l{ }_{3}\right)$. Anal. Calcd $(\%)$ for $\mathrm{C}_{30} \mathrm{H}_{39} \mathrm{BCl}_{2} \mathrm{~N}_{2} \mathrm{Si}\left(M_{\mathrm{r}}=537.45\right)$ : C, 67.04; H, 7.31; N, 5.21; Found: C, 67.36; H, 7.23; N, 5.10.

$\mathrm{LB}(\mathrm{Mes}) \mathrm{SiCl}_{2} \mathrm{Mes}(\mathbf{6})$ : To a solution of LSiMes $(378 \mathrm{mg}, 1 \mathrm{mmol})$ in toluene $(40 \mathrm{~mL})$ at room temperature, a solution of $\mathrm{MesBCl}_{2}(201$ $\mathrm{mg}, 1 \mathrm{mmol})$ in toluene $(20 \mathrm{~mL})$ was added dropwise under stirring. The mixture was stirred one day. After filtration, the colorless solution was concentrated to afford an off-white solid, which was washed with $n$-hexane $(5 \mathrm{~mL})$. Yield: $0.52 \mathrm{~g}(90 \%)$. Mp: $203{ }^{\circ} \mathrm{C}$. ${ }^{1} \mathrm{H}$ $\operatorname{NMR}\left(\mathrm{C}_{6} \mathrm{D}_{6}, 298 \mathrm{~K}, 400 \mathrm{MHz}, \mathrm{ppm}\right): \delta=8.16-8.18(\mathrm{~m}, 1 \mathrm{H}, \mathrm{Ph}-H), 7.38-7.35(\mathrm{~m}, 1 \mathrm{H}, \mathrm{Ph}-H), 7.02-6.93(\mathrm{~m}, 3 \mathrm{H}, \mathrm{Ph}-H), 6.89(\mathrm{~s}, 1 \mathrm{H}$, $\left.\mathrm{C}_{6} \mathrm{H}_{2}\right), 6.80\left(\mathrm{~s}, 1 \mathrm{H}, \mathrm{C}_{6} \mathrm{H}_{2}\right), 6.77$ (s, $2 \mathrm{H}, \mathrm{C}_{6} \mathrm{H}_{2}$ ), 2.94 (s, $\left.6 \mathrm{H}, \mathrm{Ar}-\mathrm{Me}\right), 2.92$ (s, $\left.3 \mathrm{H}, \mathrm{Ar}-\mathrm{Me}\right), 2.84$ (s, $\left.3 \mathrm{H}, \mathrm{Ar}-\mathrm{Me}\right), 2.17(\mathrm{~s}, 3 \mathrm{H}, \mathrm{Ar}-\mathrm{Me})$, 
2.03 (s, $3 \mathrm{H}, \mathrm{Ar}-\mathrm{Me}), 1.15$ (s, $\left.18 \mathrm{H}, \mathrm{CMe}_{3}\right) .{ }^{13} \mathrm{C}\left\{{ }^{1} \mathrm{H}\right\} \mathrm{NMR}\left(\mathrm{C}_{6} \mathrm{D}_{6}, 298 \mathrm{~K}, 125 \mathrm{MHz}, \mathrm{ppm}\right): \delta=166.3\left(\mathrm{PhC}(\mathrm{N} t \mathrm{Bu})_{2}\right), 145.5,144.6,144.5$, 139.7, 136.8, 135.1, 133.4, 131.0, 130.4, 130.1, 129.7, 129.3, 129.0, 128.6, 128.5, 128.4, 127.3, $125.7(\mathrm{Ph}-\mathrm{C}$ and Ar-C), $55.3(\mathrm{NCMe})$ ), $31.7\left(\mathrm{NCMe}_{3}\right), 30.6$ (Ar-Me), 26.9 (Ar-Me), 23.6 (Ar-Me), 20.9 (Ar-Me). ${ }^{29} \mathrm{Si} \mathrm{NMR}\left(\mathrm{C}_{6} \mathrm{D}_{6}, 298 \mathrm{~K}, 79 \mathrm{MHz}, \mathrm{ppm}\right): \delta=25.5$ (br, SiBr $\left.2 \mathrm{Mes}\right)$. ${ }^{11} \mathrm{~B}\left\{{ }^{1} \mathrm{H}\right\} \operatorname{NMR}\left(\mathrm{C}_{6} \mathrm{D}_{6}, 298 \mathrm{~K}, 128 \mathrm{MHz}, \mathrm{ppm}\right): \delta=5.7\left(\mathrm{br}, B \mathrm{BSiCl}_{3}\right)$. Anal. Calcd (\%) for $\mathrm{C}_{33} \mathrm{H}_{45} \mathrm{BCl}_{2} \mathrm{~N}_{2} \mathrm{Si}\left(M_{\mathrm{r}}=579.53\right): \mathrm{C}, 68.39 ; \mathrm{H}, 7.83$; $\mathrm{N}, 4.83$; Found: C, 68.52; $\mathrm{H}, 7.58 ; \mathrm{N}, 4.65$.

$\mathrm{LB}(\mathrm{Mes}) \mathrm{SiBr}_{2} \mathrm{Mes}(7)$ : To a solution of LSiMes (378 mg, $\left.1 \mathrm{mmol}\right)$ in toluene $(40 \mathrm{~mL})$ at $-78{ }^{\circ} \mathrm{C}$, a solution of $\mathrm{MesBBr}_{2}(290 \mathrm{mg}, 1 \mathrm{mmol})$ in toluene $(20 \mathrm{~mL})$ was added dropwise under stirring. The mixture was slowly warmed to room temperature and stirred overnight. After filtration, the colorless solution was concentrated to ca. $5 \mathrm{~mL}$. The solution was cooled to $-26{ }^{\circ} \mathrm{C}$ for $12 \mathrm{~h}$ to afford colorless crystals which were suitable for X-ray diffraction study. Yield: $0.60 \mathrm{~g}(90 \%)$. Mp: $251{ }^{\circ} \mathrm{C} .{ }^{1} \mathrm{H}$ NMR $\left(\mathrm{THF}-\mathrm{D}_{8}, 298 \mathrm{~K}, 300 \mathrm{MHz}, \mathrm{ppm}\right): \delta=$ 8.40-7.10 (m, $5 \mathrm{H}, \mathrm{Ph}-\mathrm{H}), 6.88\left(\mathrm{~s}, 2 \mathrm{H}, \mathrm{C}_{6} \mathrm{H}_{2}\right), 6.83\left(\mathrm{~s}, 1 \mathrm{H}, \mathrm{C}_{6} \mathrm{H}_{2}\right), 6.82\left(\mathrm{~s}, 1 \mathrm{H}, \mathrm{C}_{6} \mathrm{H}_{2}\right), 2.90(\mathrm{~s}, 3 \mathrm{H}, \mathrm{Ar}-\mathrm{Me}), 2.80(\mathrm{~s}, 6 \mathrm{H}, \mathrm{Ar}-\mathrm{Me}), 2.73(\mathrm{~s}$, $3 \mathrm{H}, \mathrm{Ar}-\mathrm{Me}$ ), 2.27 (s, $3 \mathrm{H}, \mathrm{Ar}-\mathrm{Me}$ ), 2.26 (s, $3 \mathrm{H}, \mathrm{Ar}-\mathrm{Me}$ ), 1.19 (s, $\left.18 \mathrm{H}, \mathrm{CMe}_{3}\right) .{ }^{13} \mathrm{C}\left\{{ }^{1} \mathrm{H}\right\} \mathrm{NMR}\left(\mathrm{THF}-\mathrm{D}_{8}, 298 \mathrm{~K}, 75 \mathrm{MHz}, \mathrm{ppm}\right): \delta=167.0$ $\left(\mathrm{PhC}(\mathrm{N} t \mathrm{Bu})_{2}\right), 145.0,144.0,143.9,139.3,136.2,134.0,132.8,130.3,130.2,129.7,129.5,128.8,128.7,128.1,127.9,127.3(\mathrm{Ph}-\mathrm{C}$

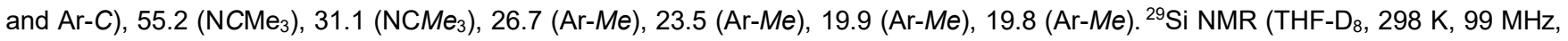
ppm): $\delta=24.0$ (br, SiBr 2 Mes). ${ }^{11} \mathrm{~B}\left\{{ }^{1} \mathrm{H}\right\}$ NMR (THF-D $\left.8,298 \mathrm{~K}, 160.5 \mathrm{MHz}, \mathrm{ppm}\right): \delta=5.8$ (br, BSiCl $\left.{ }_{3}\right)$. Anal. Calcd (\%) for $\mathrm{C}_{33} \mathrm{H}_{45} \mathrm{BBr}_{2} \mathrm{~N}_{2} \mathrm{Si}\left(M_{\mathrm{r}}=668.44\right)$ : C, 59.30; H, 6.79; N, 4.19; Found: C, 59.55; H, 6.73; N, 4.26. 

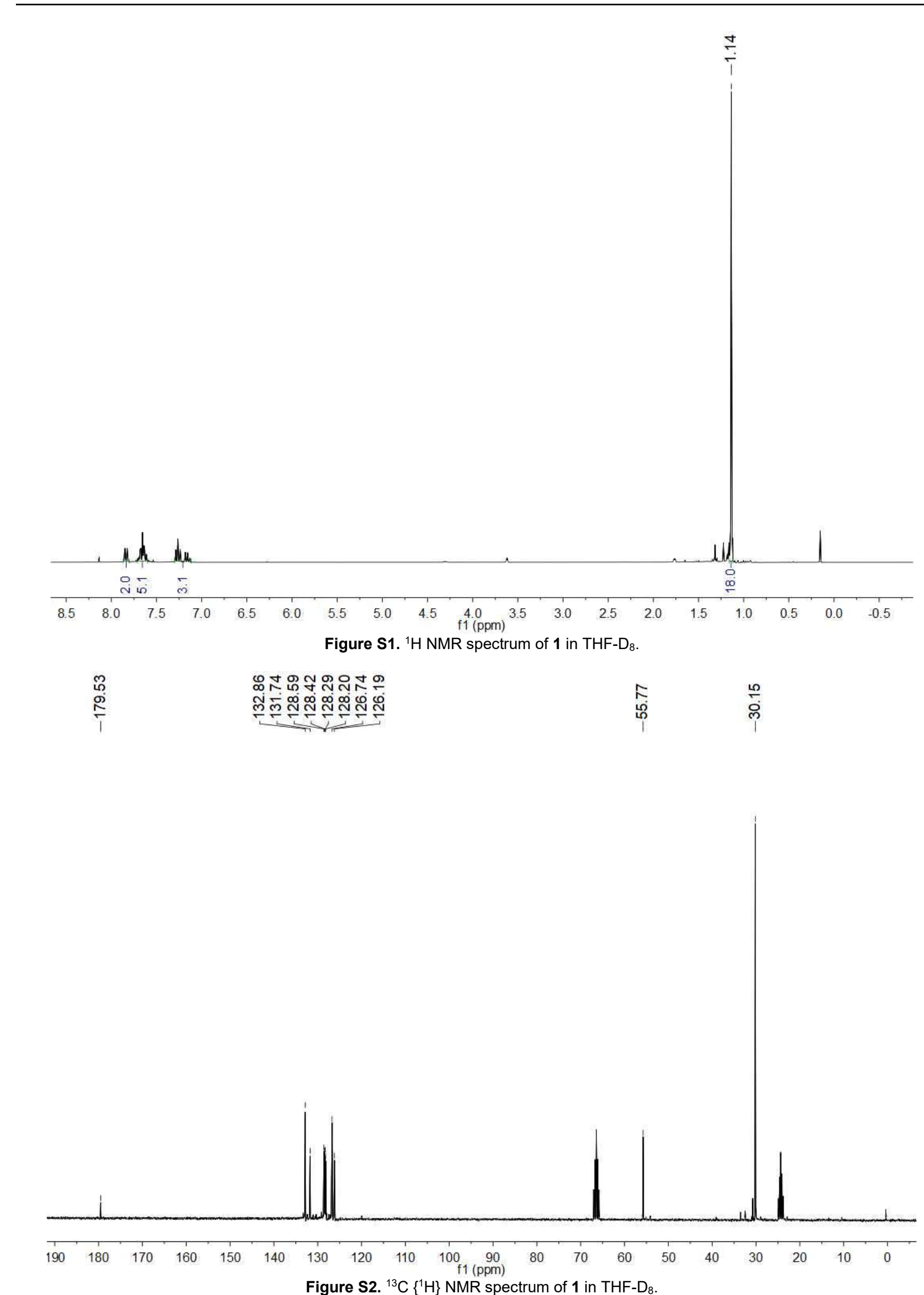

Figure S2. ${ }^{13} \mathrm{C}\left\{{ }^{1} \mathrm{H}\right\}$ NMR spectrum of 1 in THF- $\mathrm{D}_{8}$. 


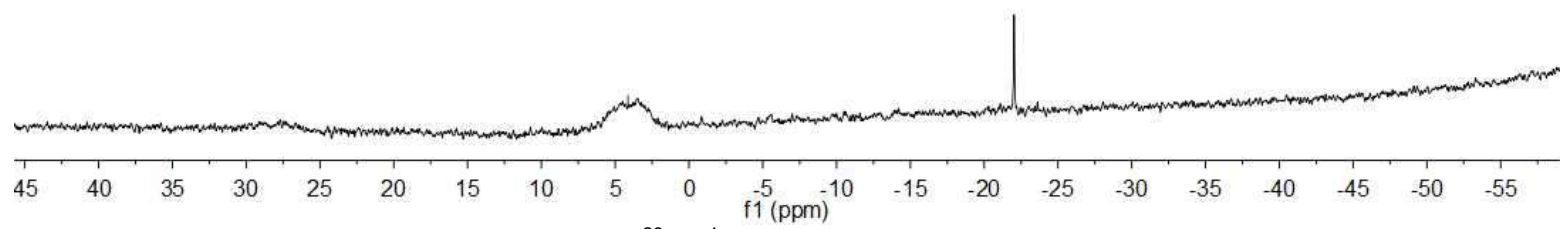

Figure S3. ${ }^{29} \mathrm{Si}\left\{{ }^{1} \mathrm{H}\right\}$ spectrum of 1 in THF-D 8 .

ஜำ

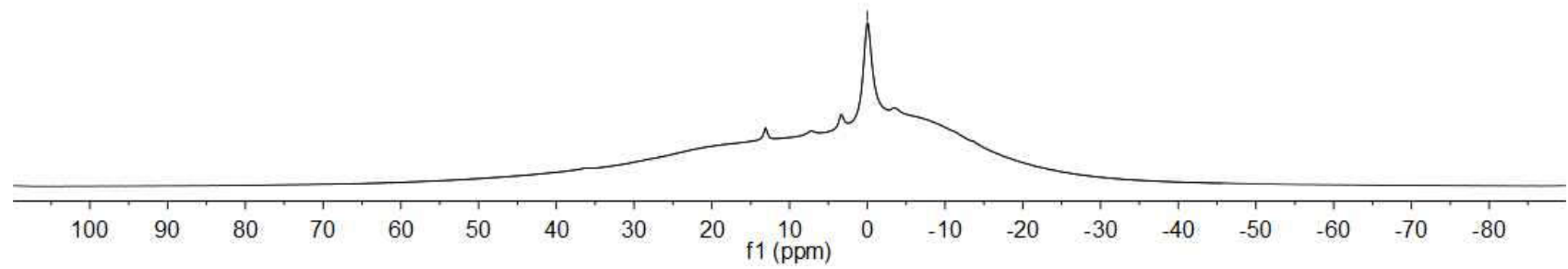

Figure S4. ${ }^{11} \mathrm{~B}\left\{{ }^{1} \mathrm{H}\right\}$ spectrum of 1 in THF- $\mathrm{D}_{8}$. 


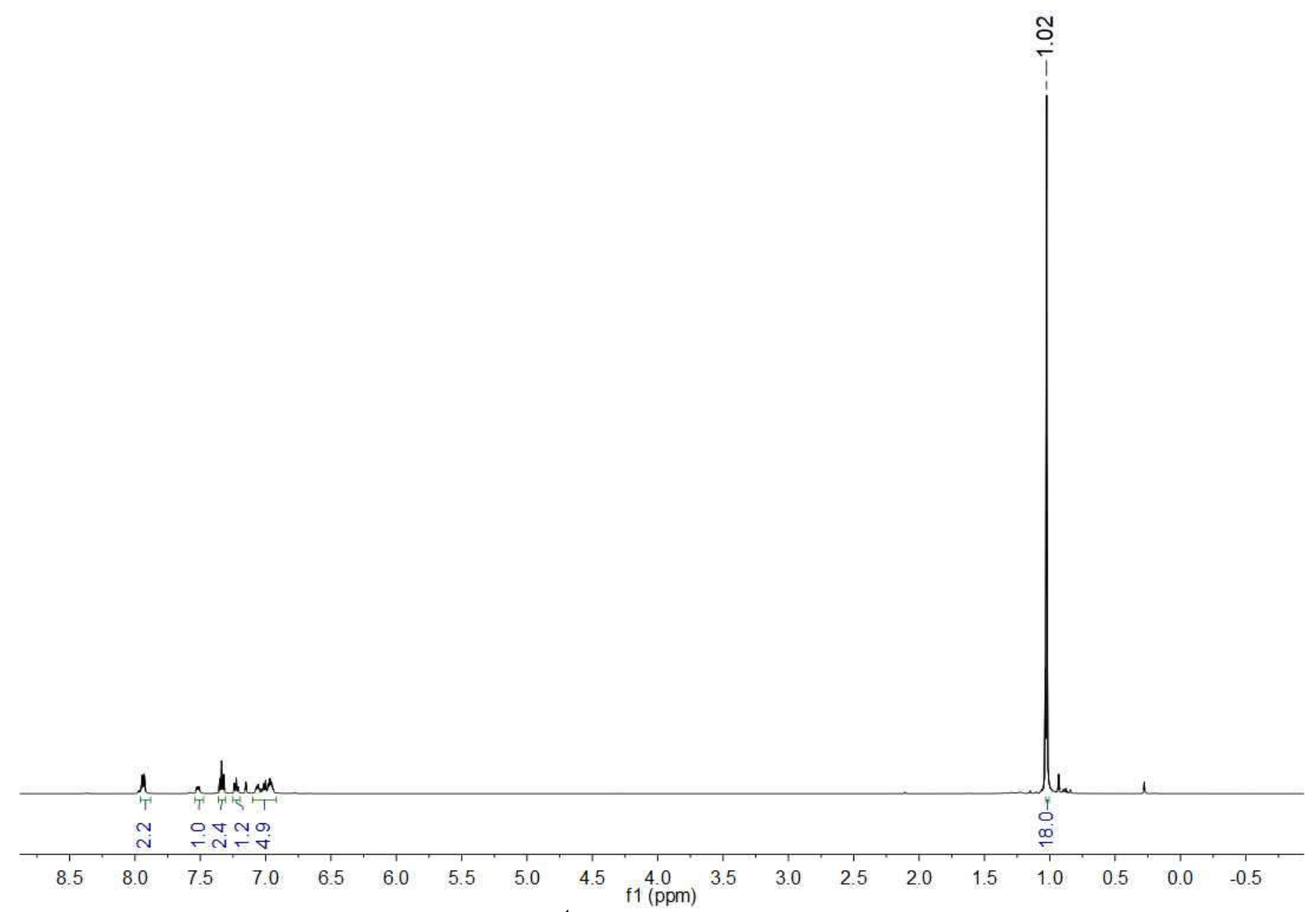

Figure S5. ${ }^{1} \mathrm{H}$ NMR spectrum of 2 in $\mathrm{C}_{6} \mathrm{D}_{6}$.

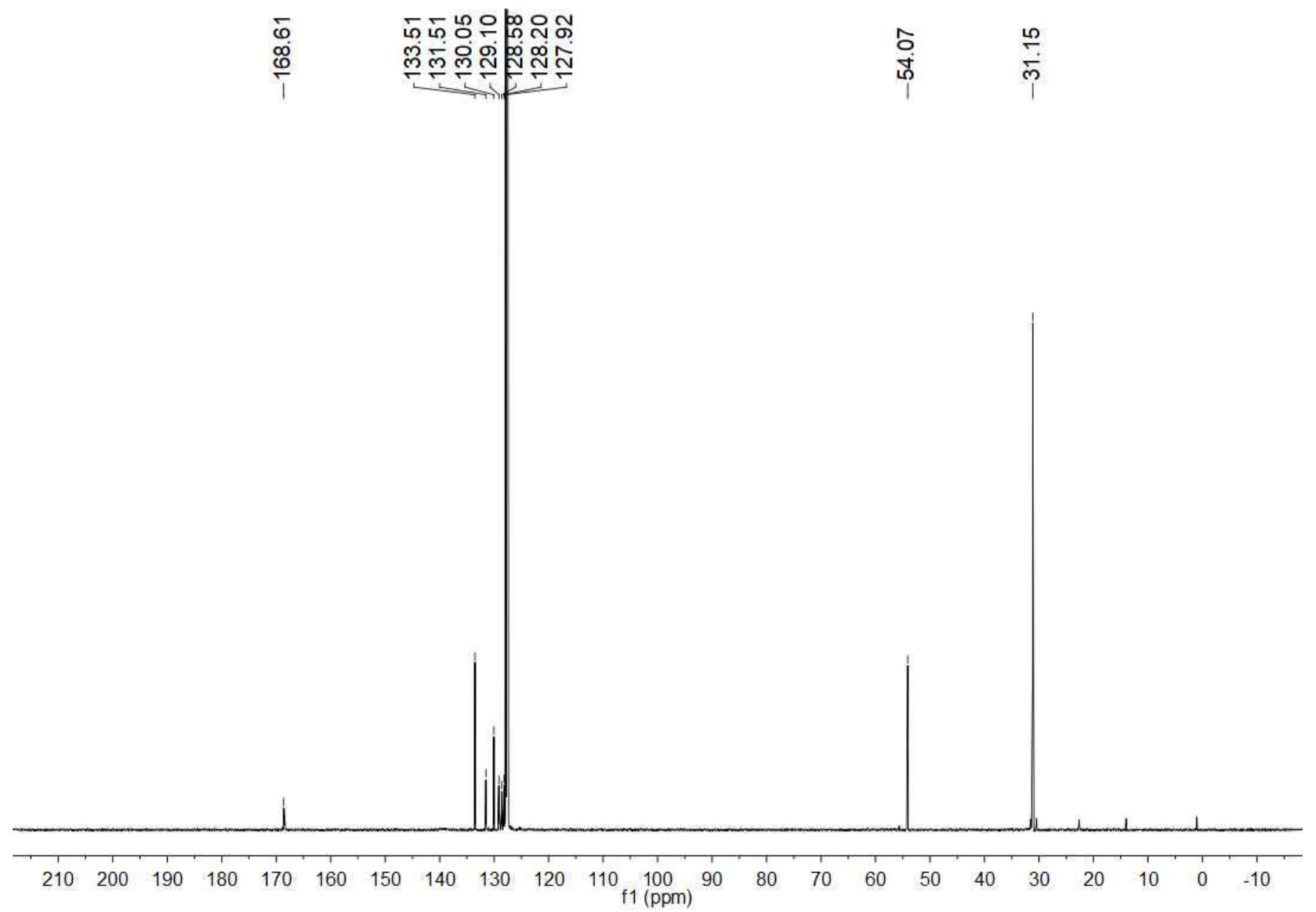

Figure S6. ${ }^{13} \mathrm{C}\left\{{ }^{1} \mathrm{H}\right\}$ NMR spectrum of 2 in $\mathrm{C}_{6} \mathrm{D}_{6}$. 


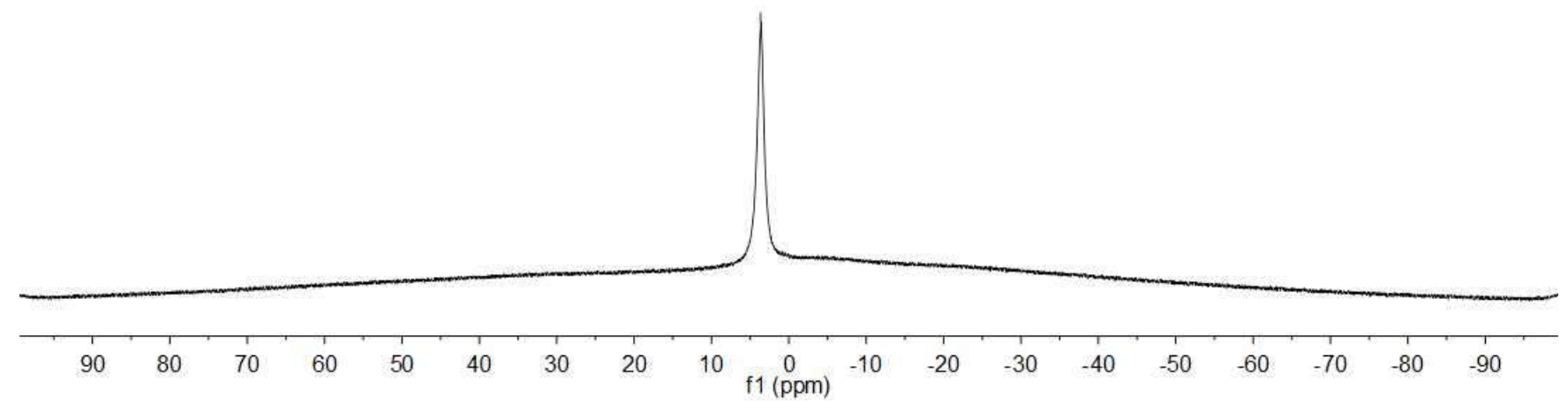

Figure S7. ${ }^{11} \mathrm{~B}\left\{{ }^{1} \mathrm{H}\right\}$ spectrum of 2 in $\mathrm{C}_{6} \mathrm{D}_{6}$.

\begin{tabular}{|c|c|}
\hline $\begin{array}{l}20 \\
\infty \\
00 \\
0 \\
1\end{array}$ & Sָ̃ \\
\hline
\end{tabular}

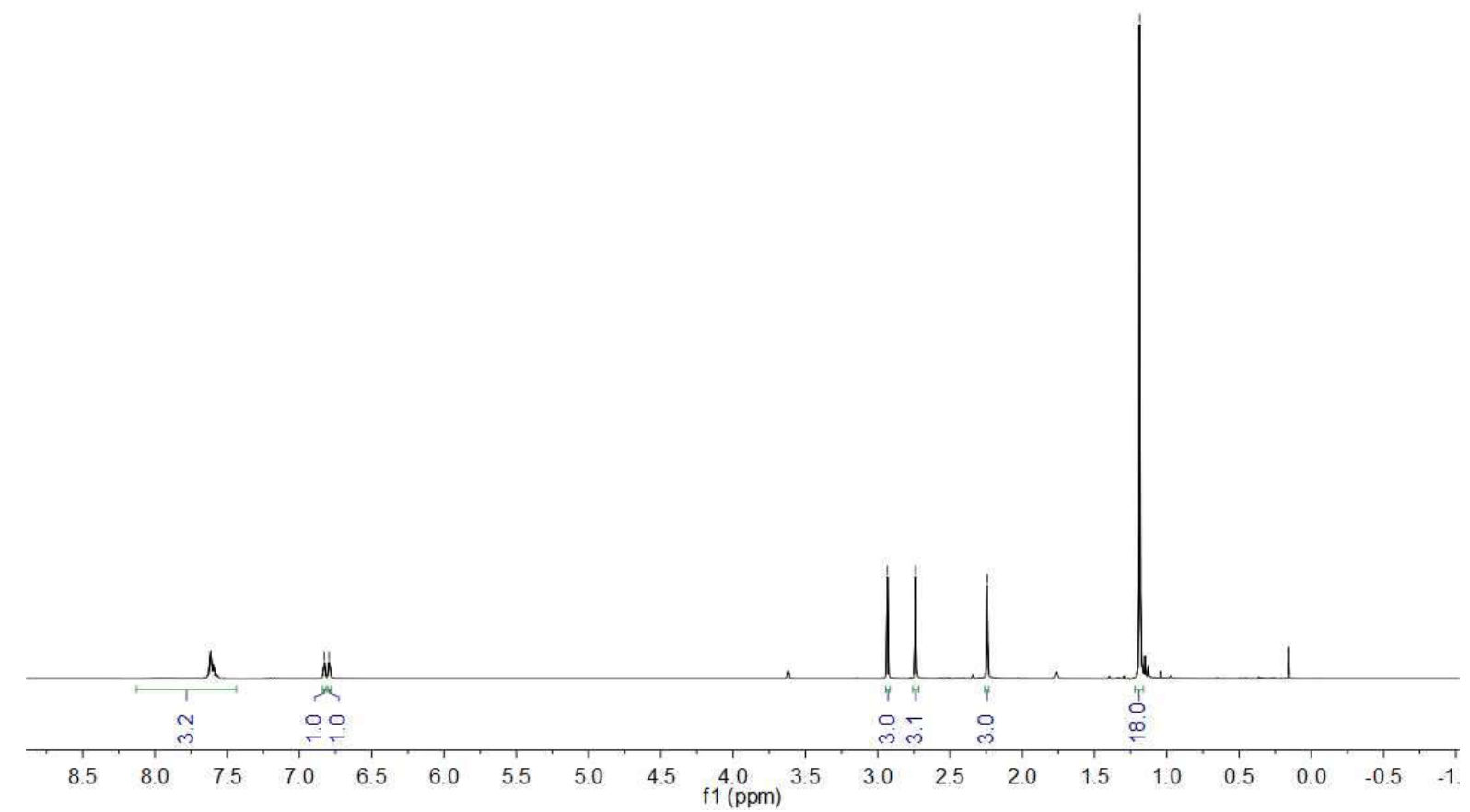

Figure S8. $1 \mathrm{H}$ NMR spectrum of 3 in THF-D. 


$\begin{array}{ll}0 & 0 \\ 0 & 0\end{array}$

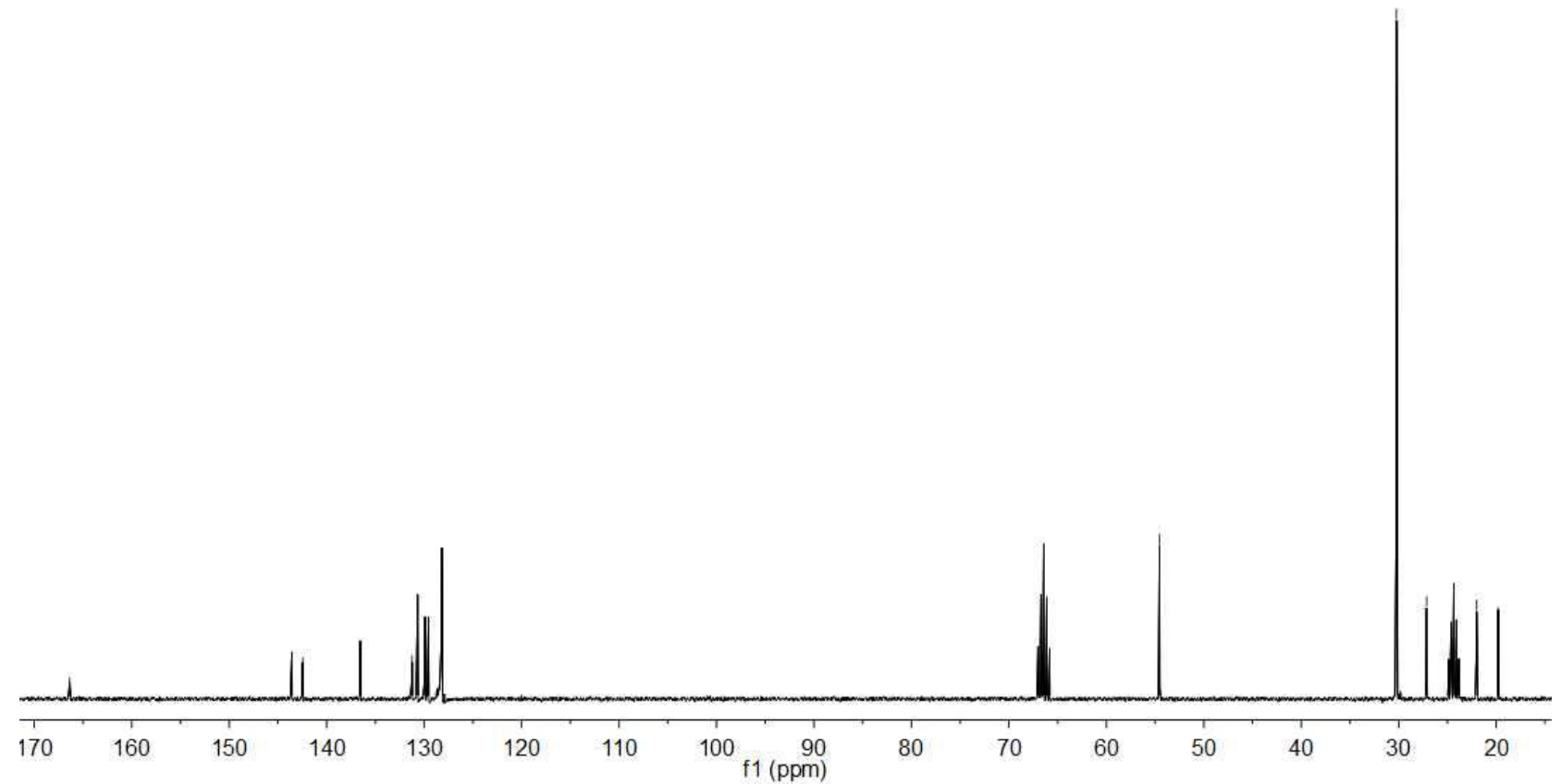

Figure S9. ${ }^{13} \mathrm{C}\left\{{ }^{1} \mathrm{H}\right\}$ NMR spectrum of 3 in THF-D $\mathrm{D}_{8}$.

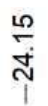

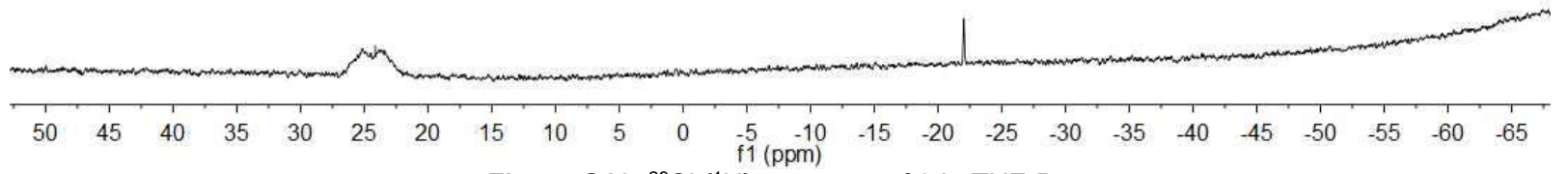

Figure S10. ${ }^{29} \mathrm{Si}\left\{{ }^{1} \mathrm{H}\right\}$ spectrum of 3 in THF-D. 


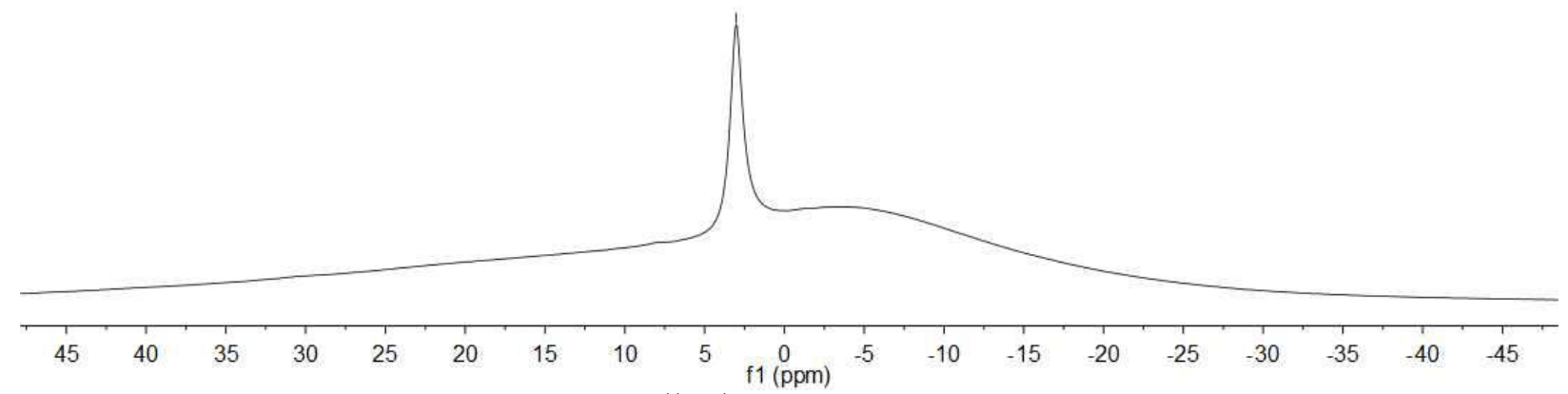

Figure S11. ${ }^{11} \mathrm{~B}\left\{{ }^{1} \mathrm{H}\right\}$ spectrum of 3 in THF- $\mathrm{D}_{8}$.

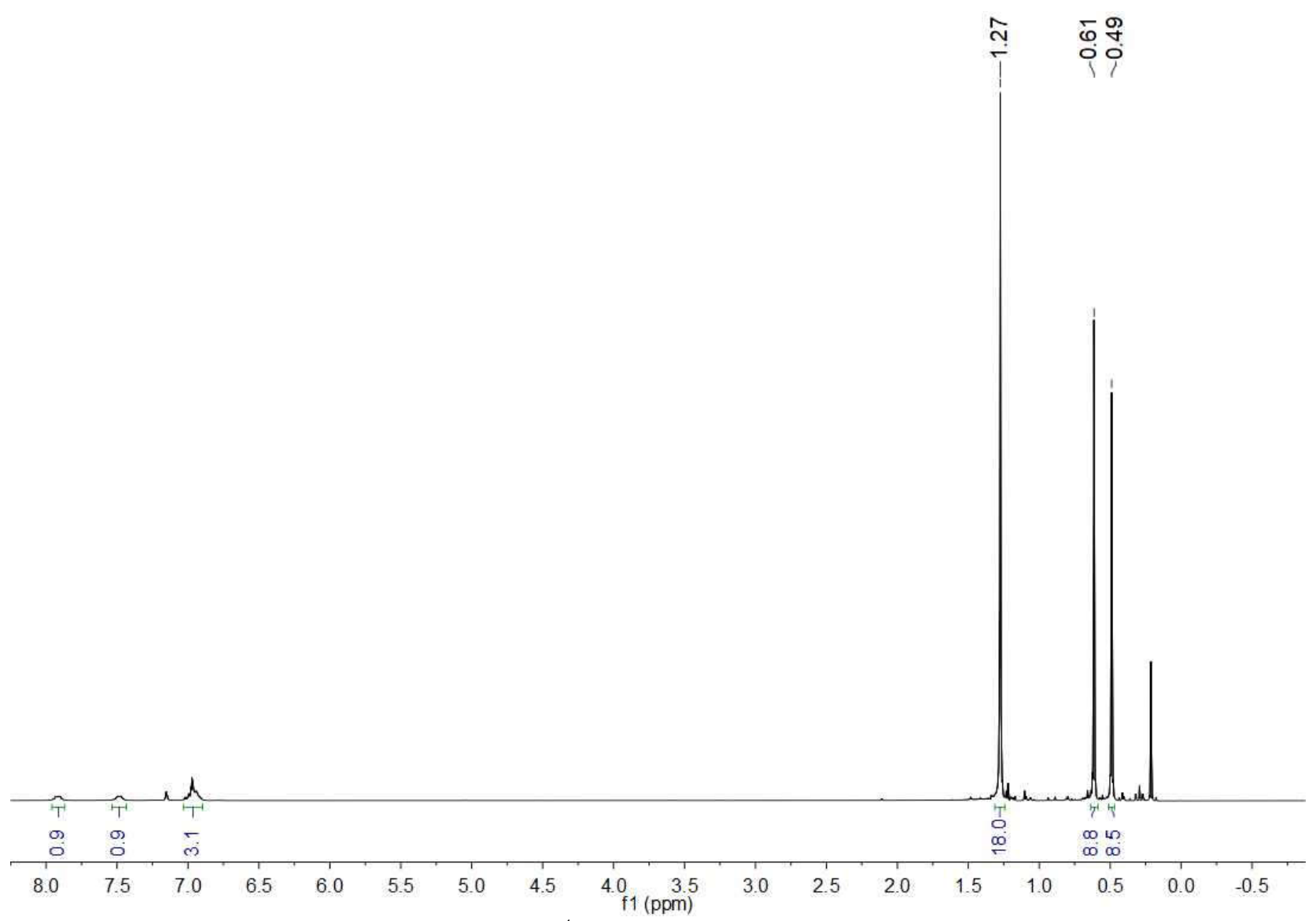

Figure S12. ${ }^{1} \mathrm{H}$ NMR spectrum of 4 in $\mathrm{C}_{6} \mathrm{D}_{6}$. 


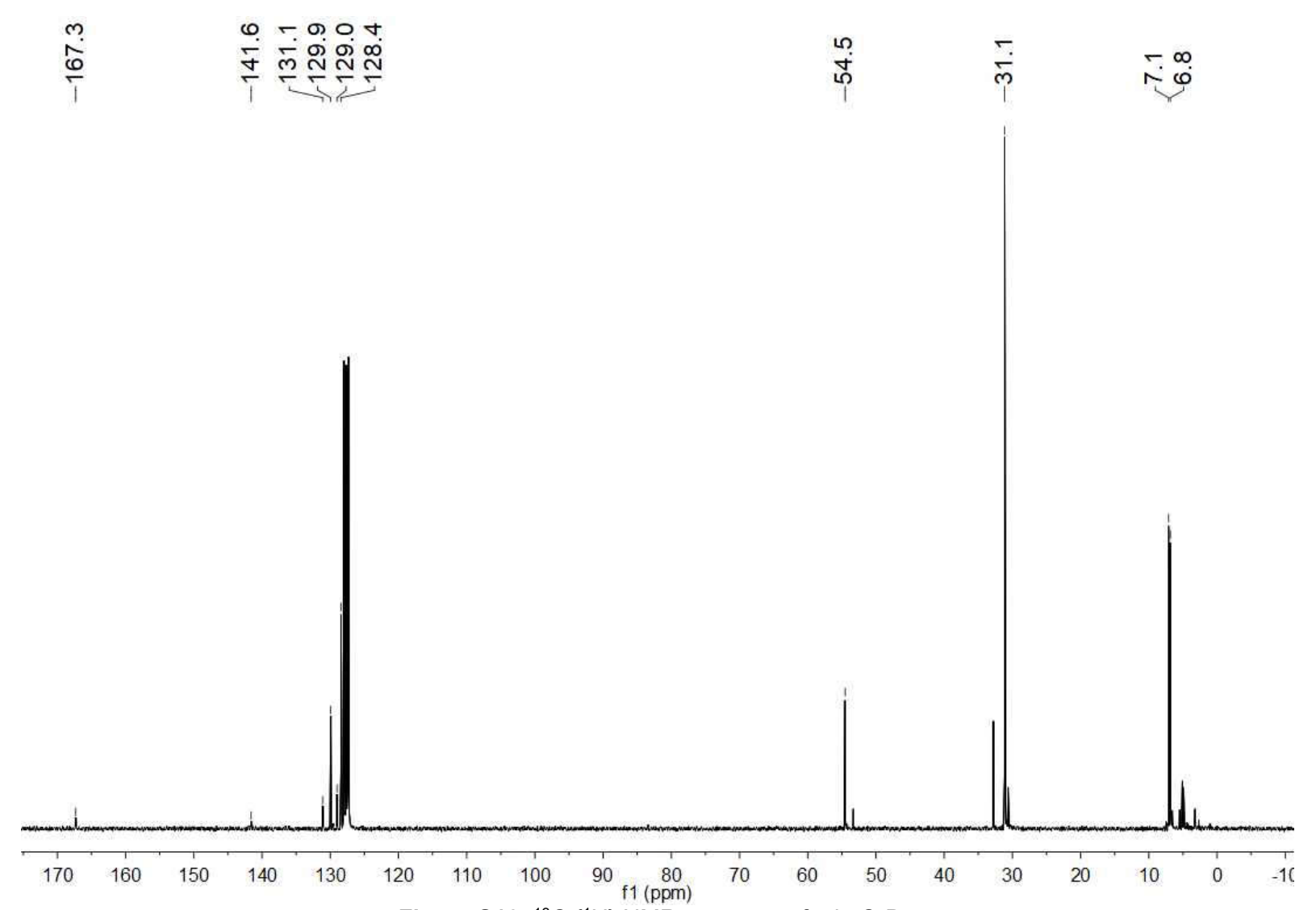

Figure $513 .{ }^{13} \mathrm{C}\left\{{ }^{1} \mathrm{H}\right\}$ NMR spectrum of 4 in $\mathrm{C}_{6} \mathrm{D}_{6}$.
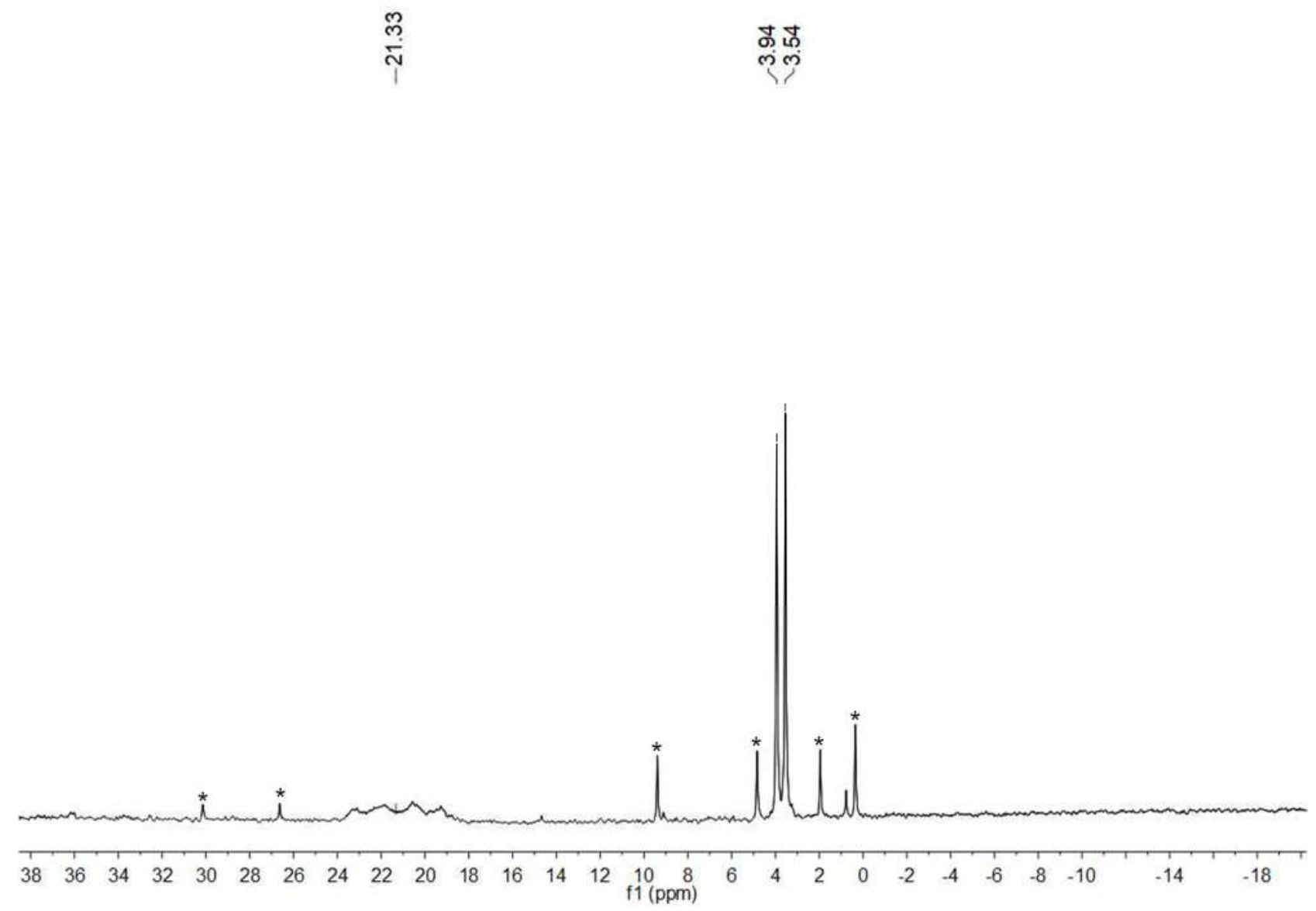

Figure S14. ${ }^{29} \mathrm{Si}\left\{{ }^{1} \mathrm{H}\right\}$ spectrum of 4 in $\mathrm{C}_{6} \mathrm{D}_{6}\left({ }^{*}\right.$ unidentified compound because of the decomposition of 4$)$. 


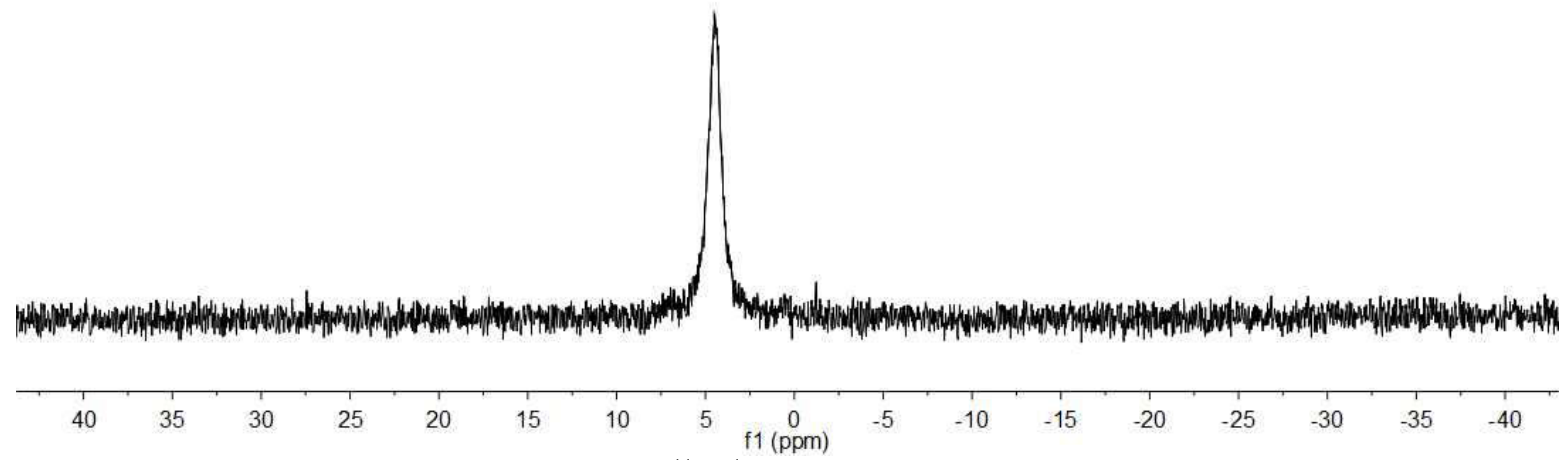

Figure S15. ${ }^{11} \mathrm{~B}\left\{{ }^{1} \mathrm{H}\right\}$ spectrum of 4 in $\mathrm{C}_{6} \mathrm{D}_{6}$.

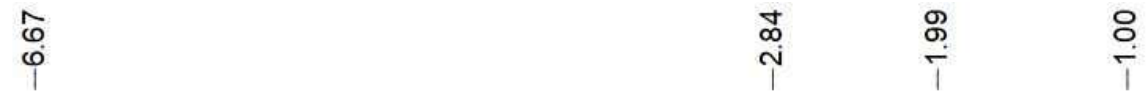

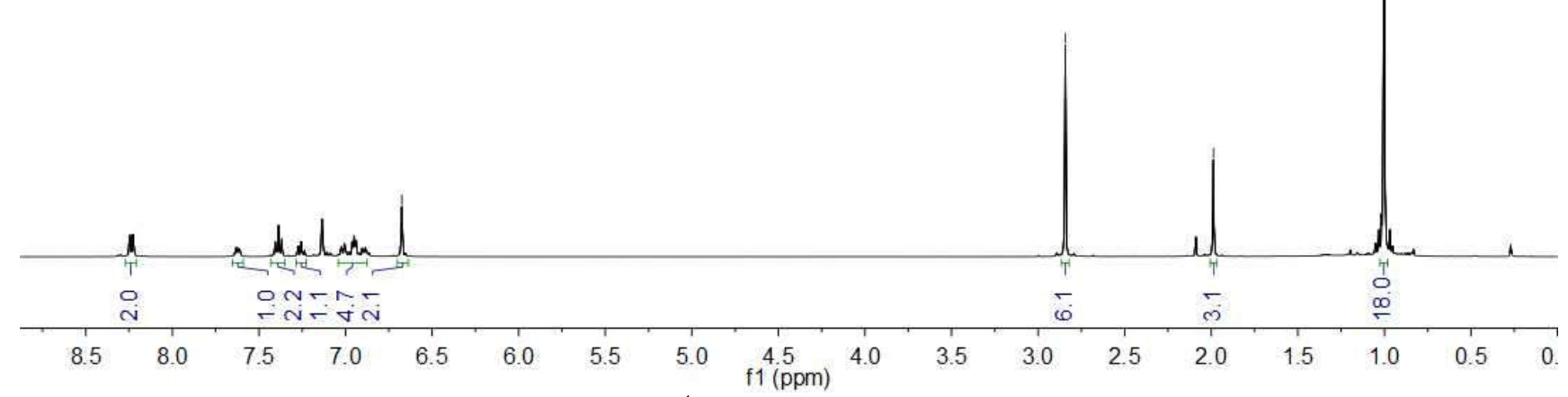

Figure S16. ${ }^{1} \mathrm{H}$ NMR spectrum of 5 in $\mathrm{C}_{6} \mathrm{D}_{6}$. 


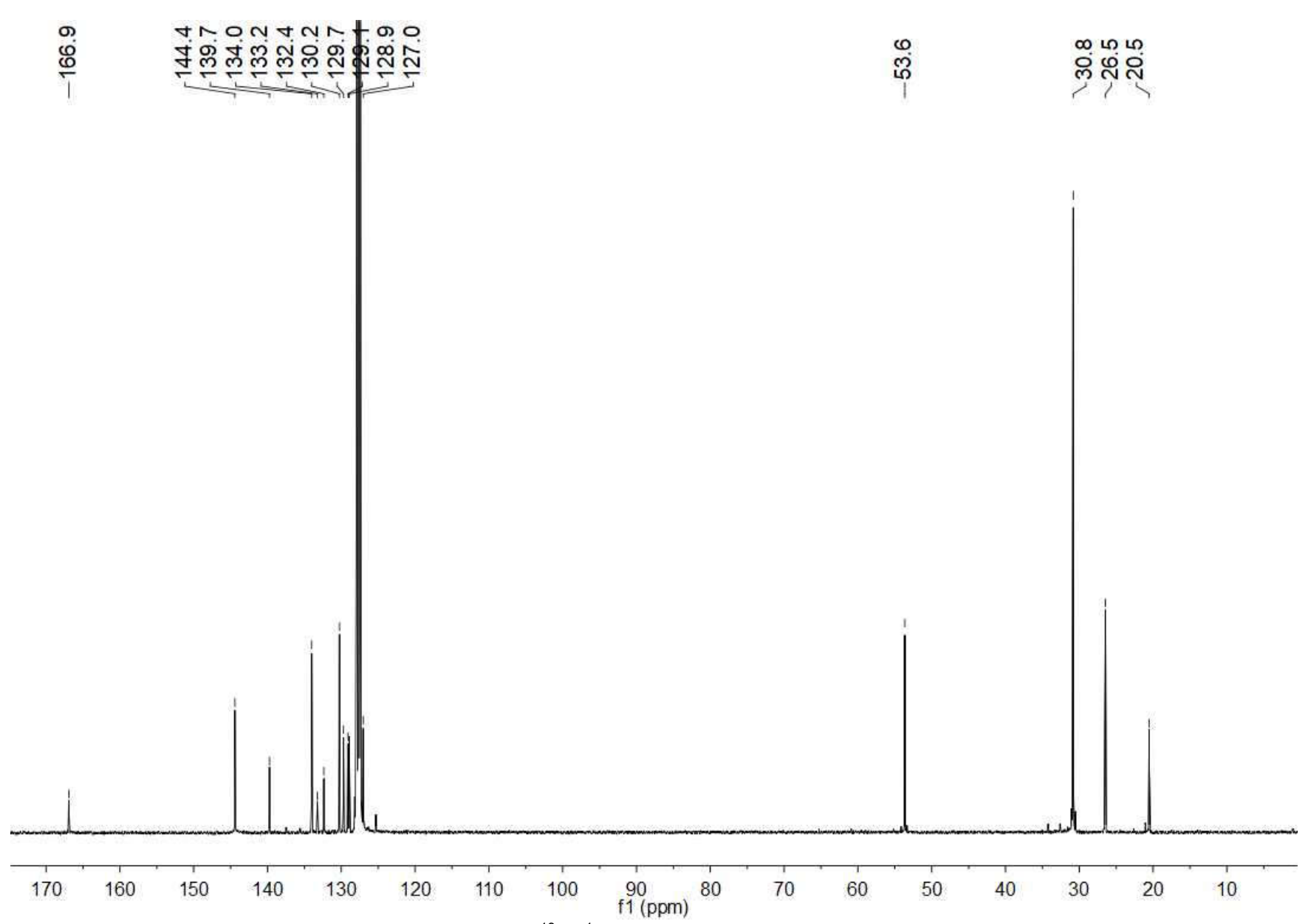

Figure $S 17 .{ }^{13} \mathrm{C}\left\{{ }^{1} \mathrm{H}\right\}$ NMR spectrum of 5 in $\mathrm{C}_{6} \mathrm{D}_{6}$.

$\stackrel{\circ}{\stackrel{\infty}{\sim}}$

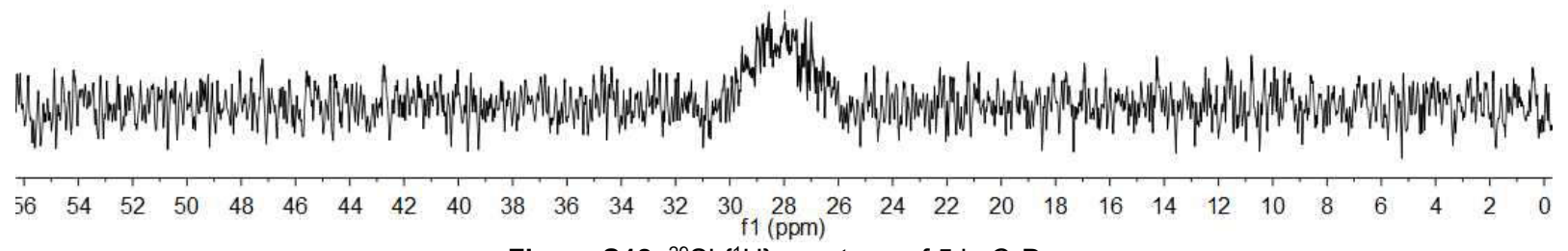

Figure S18. ${ }^{29} \mathrm{Si}\left\{{ }^{1} \mathrm{H}\right\}$ spectrum of 5 in $\mathrm{C}_{6} \mathrm{D}_{6}$. 


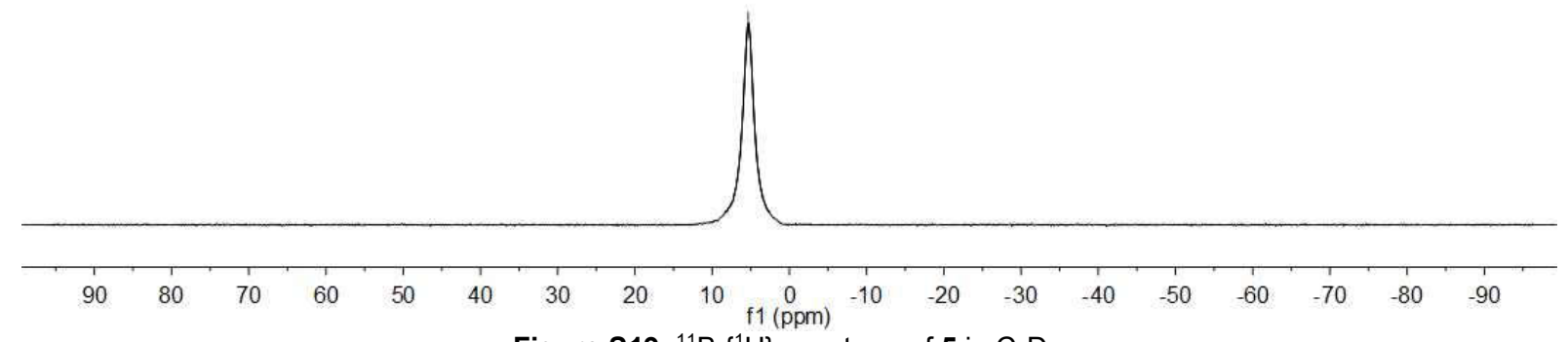

Figure S19. ${ }^{11} \mathrm{~B}\left\{{ }^{1} \mathrm{H}\right\}$ spectrum of 5 in $\mathrm{C}_{6} \mathrm{D}_{6}$.

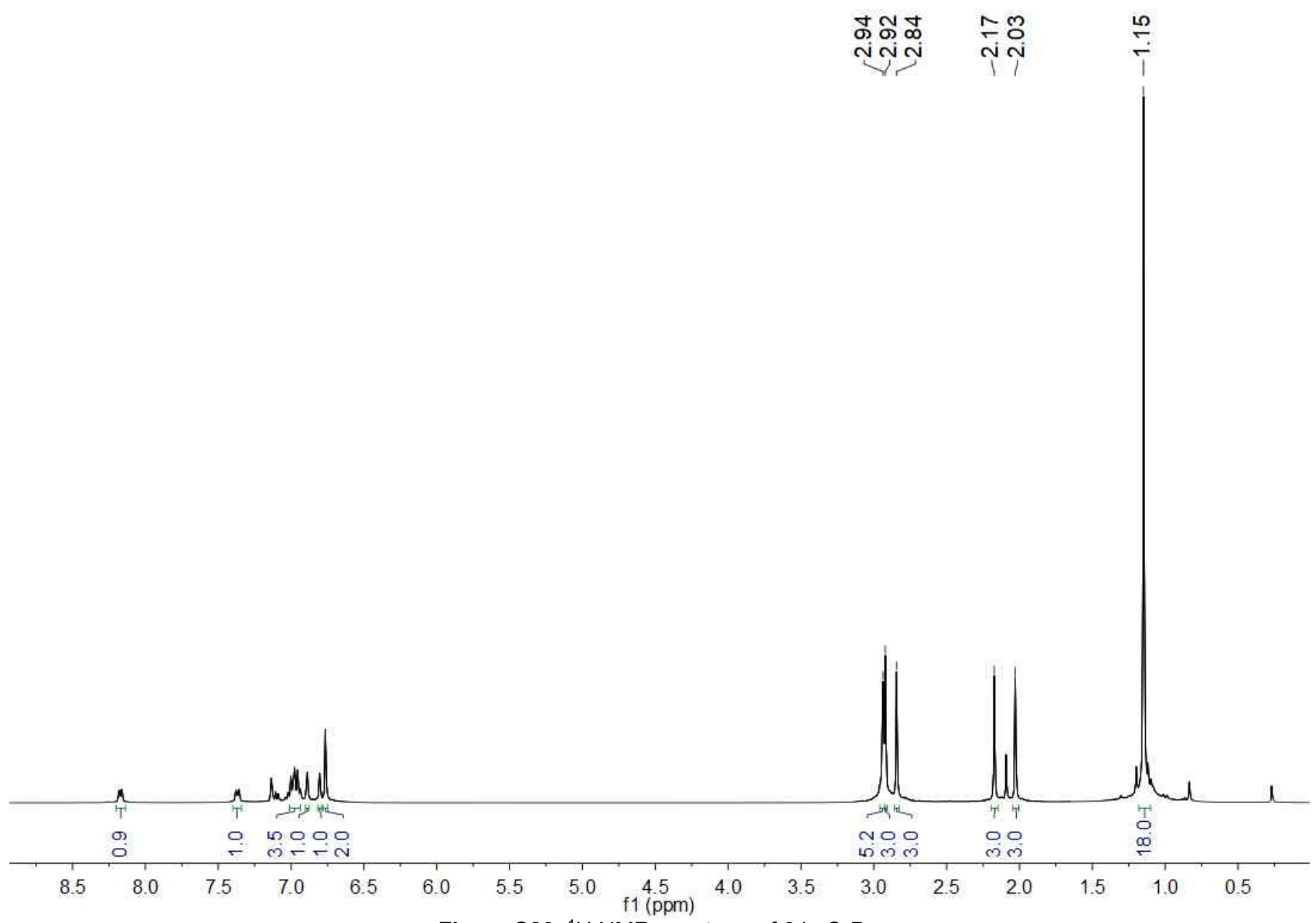

Figure S20. ${ }^{1} \mathrm{H}$ NMR spectrum of 6 in $\mathrm{C}_{6} \mathrm{D}_{6}$. 


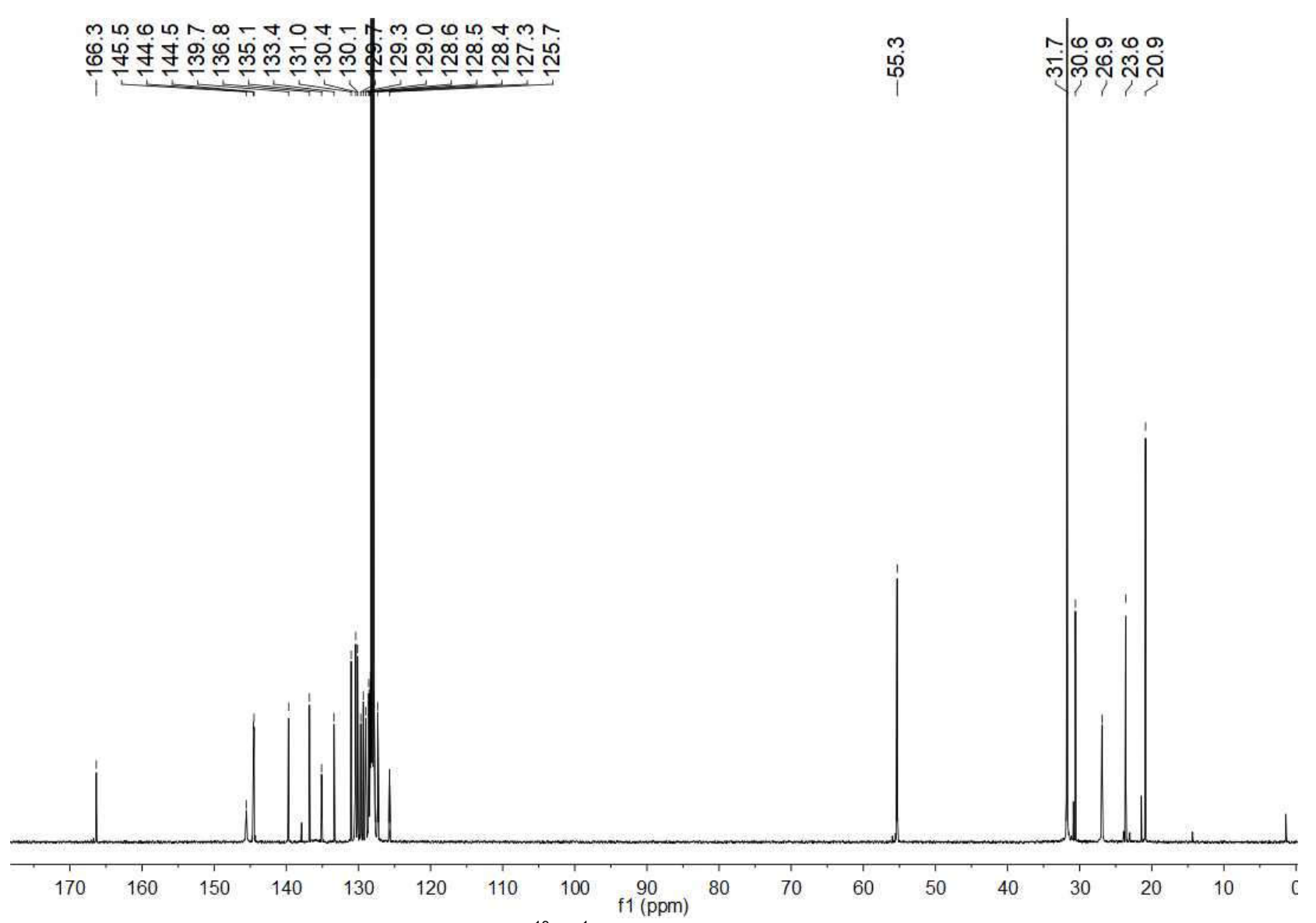

Figure S21. ${ }^{13} \mathrm{C}\left\{{ }^{1} \mathrm{H}\right\}$ NMR spectrum of 6 in $\mathrm{C}_{6} \mathrm{D}_{6}$. กั่

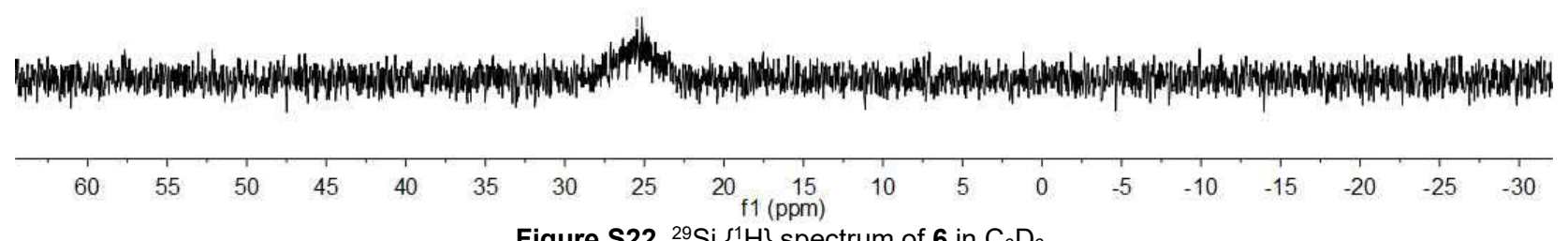

Figure S22. ${ }^{29} \mathrm{Si}\left\{{ }^{1} \mathrm{H}\right\}$ spectrum of 6 in $\mathrm{C}_{6} \mathrm{D}_{6}$. 


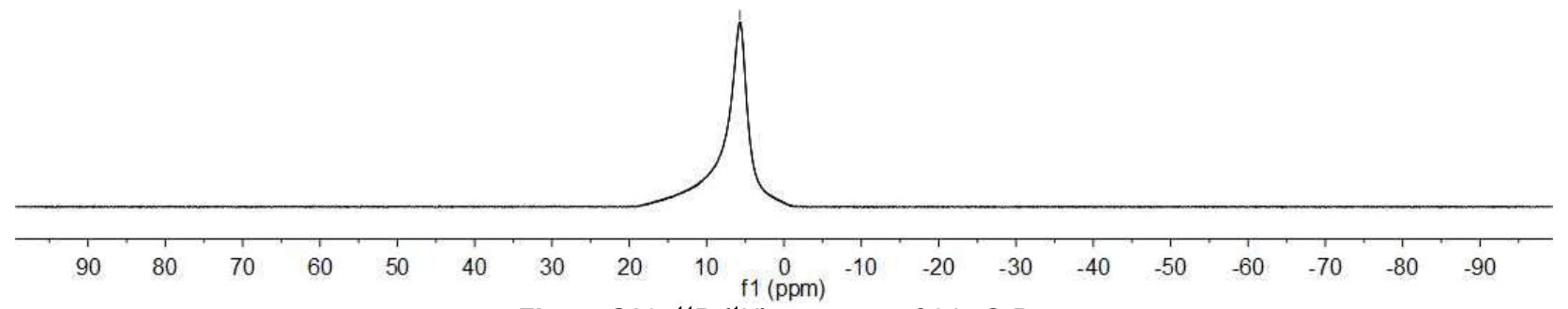

Figure S23. ${ }^{11} \mathrm{~B}\left\{{ }^{1} \mathrm{H}\right\}$ spectrum of 6 in $\mathrm{C}_{6} \mathrm{D}_{6}$.

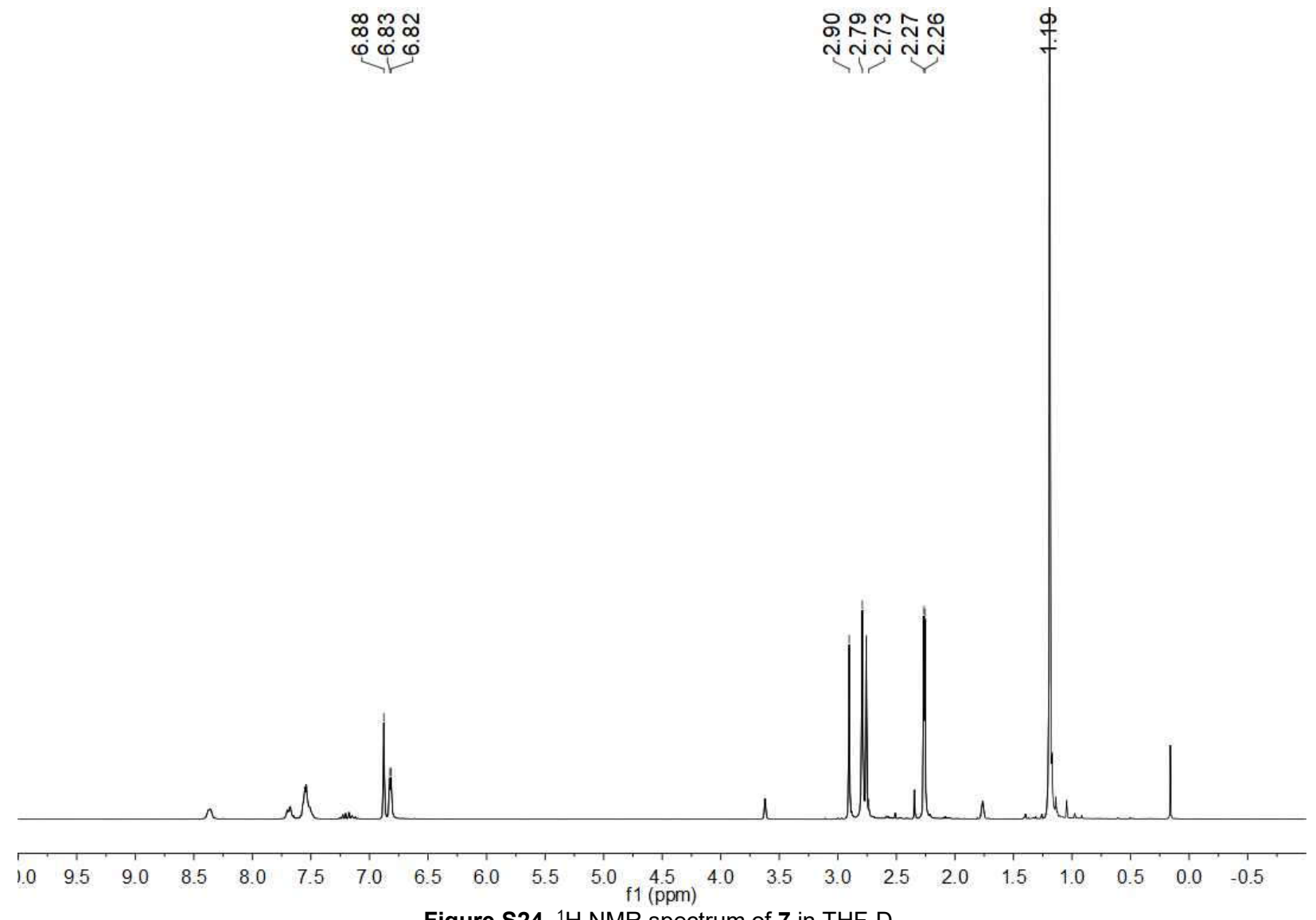

Figure S24. ${ }^{1} \mathrm{H}$ NMR spectrum of 7 in THF- $\mathrm{D}_{8}$ 


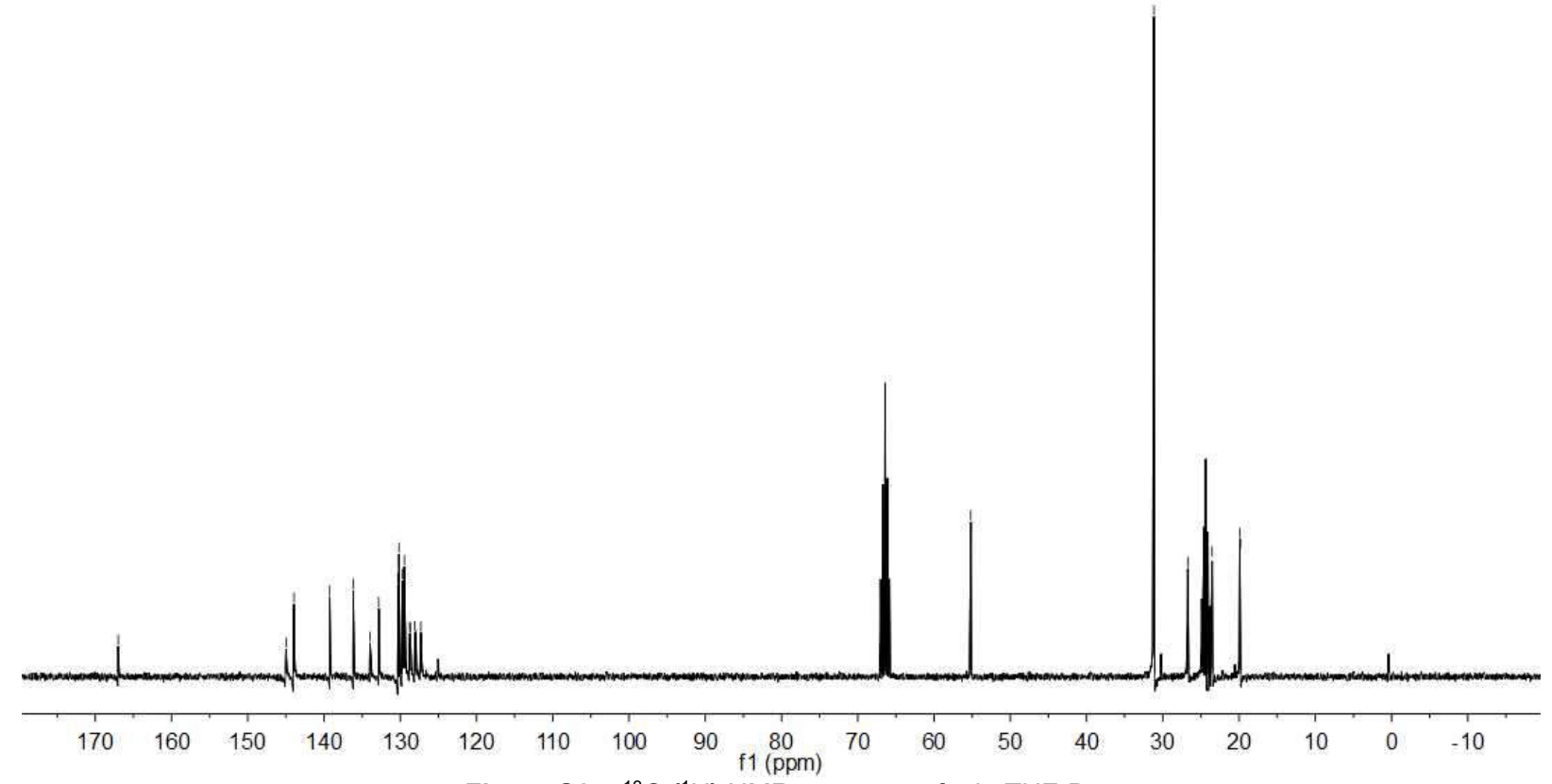

ลे

Figure S25. ${ }^{13} \mathrm{C}\left\{{ }^{1} \mathrm{H}\right\}$ NMR spectrum of 7 in THF-D 8

พิ

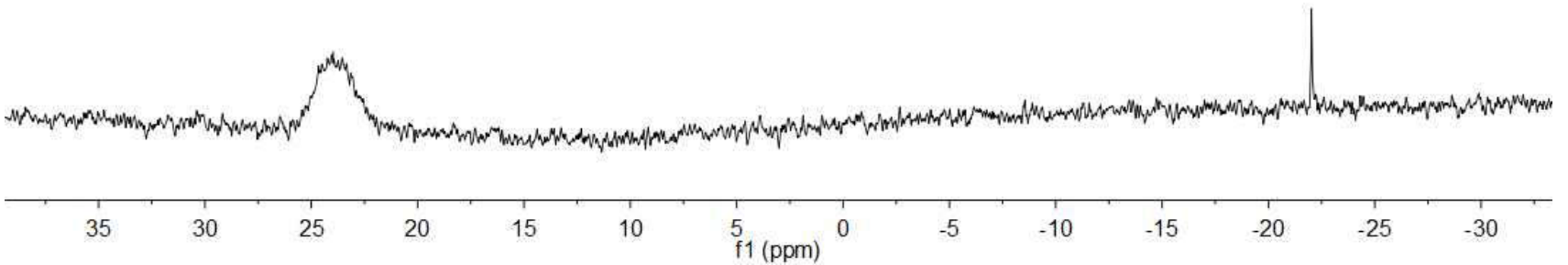

Figure S26. ${ }^{29} \mathrm{Si}\left\{{ }^{1} \mathrm{H}\right\}$ spectrum of 7 in THF-D 8 


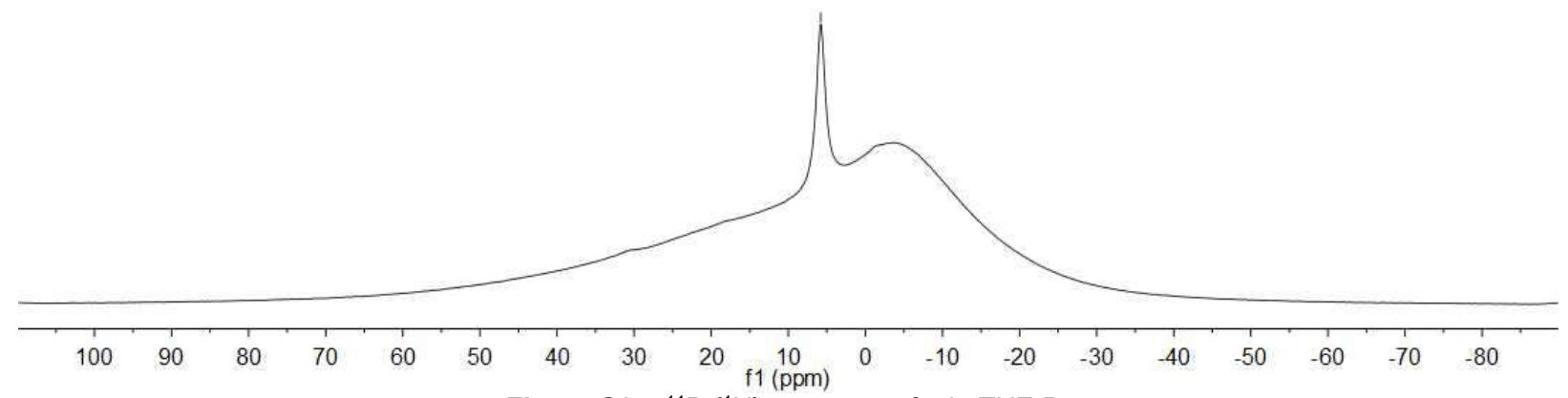

Figure S27. ${ }^{11} \mathrm{~B}\left\{{ }^{1} \mathrm{H}\right\}$ spectrum of 7 in THF- $\mathrm{D}_{8}$ 
2. The Proposed Reaction Mechanism.

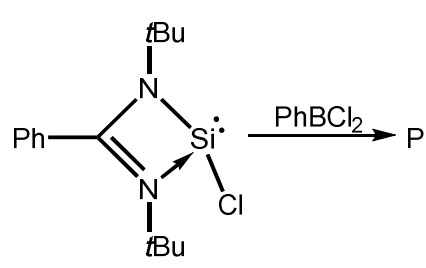

tBu

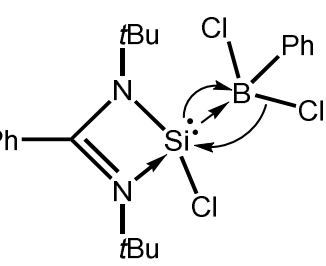

1<smiles></smiles>

2

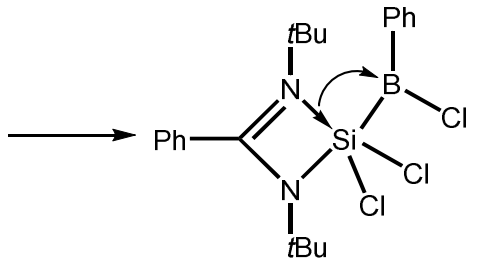

A

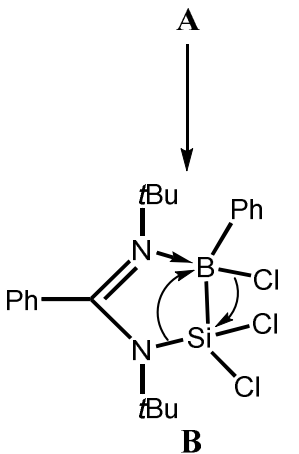

Scheme S1 The proposed mechanism for the formation of compound 2. 


\section{X-Ray Crystallographic Analysis}

The data for 1, 3, and $\mathbf{7}$ were collected from shock-cooled crystals at 100(2) K, on a BRUKER D8 three circle diffractometer equipped with an INCOATEC Ag Microsource with mirror optics $\left(\mathrm{AgK}_{\mathrm{a}}\right.$ radiation, $\lambda=0.56086 \AA$ ) or an INCOATEC Mo Microsource with mirror optics (MoK ${ }_{\alpha}$ radiation, $\lambda=0.71073 \AA$ ). The data for 2 were collected on a BRUKER FRAMBO diffractometer (CuK $\mathrm{K}_{\alpha}$ radiation, $\lambda=1.54178 \AA$ ) at $173(2) \mathrm{K}$. The data were integrated with SAINT. ${ }^{5}$ A multi-scan absorption correction was applied using SADABS. ${ }^{6}$ The structures were solved by SHELXT' and refined on $F^{2}$ using SHELXL ${ }^{8}$ in the graphical user interface SHELXLE. ${ }^{9}$

Table S1. Crystal data and refinements

\begin{tabular}{|c|c|c|c|c|}
\hline Compound & 1 & 2 & 3 & 7 \\
\hline CCDC number & 1971839 & 1971840 & 1971841 & 1971842 \\
\hline Empirical formula & $\mathrm{C}_{21} \mathrm{H}_{28} \mathrm{BCl}_{3} \mathrm{~N}_{2} \mathrm{Si}$ & $\mathrm{C}_{21} \mathrm{H}_{28} \mathrm{BCl}_{3} \mathrm{~N}_{2} \mathrm{Si}$ & $\mathrm{C}_{24} \mathrm{H}_{34} \mathrm{BCl}_{3} \mathrm{~N}_{2} \mathrm{Si}$ & $\mathrm{C}_{33} \mathrm{H}_{45} \mathrm{BBr}_{2} \mathrm{~N}_{2} \mathrm{Si}$ \\
\hline $\begin{array}{l}\text { Formula weight } \\
\left(\mathrm{g} \mathrm{mol}^{-1}\right)\end{array}$ & 453.70 & 453.70 & 495.78 & 668.43 \\
\hline Temperature $(\mathrm{K})$ & $100(2)$ & $173(2)$ & $100(2)$ & $100(2)$ \\
\hline Wavelength $(\AA)$ & 0.71073 & 1.54178 & 0.56086 & 0.56086 \\
\hline Crystal system & Orthorhombic & Orthorhombic & Monoclinic & Monoclinic \\
\hline Space group & Pnma & $P 212{ }_{1}{ }_{1}$ & $P 2_{1} / \mathrm{n}$ & $P 2_{1} / \mathrm{n}$ \\
\hline a $(\AA)$ & $18.033(3)$ & $8.892(2)$ & $9.929(2)$ & $8.942(2)$ \\
\hline$b(\AA)$ & $10.585(2)$ & $10.730(2)$ & 17.966(3) & $17.193(2)$ \\
\hline$c(\AA)$ & $12.010(2)$ & $25.025(3)$ & $14.202(2)$ & $21.218(3)$ \\
\hline$a\left({ }^{\circ}\right)$ & 90 & 90 & 90 & 90 \\
\hline $\mathrm{b}\left({ }^{\circ}\right)$ & 90 & 90 & $92.27(2)$ & $99.52(3)$ \\
\hline$g\left(\left(^{\circ}\right)\right.$ & 90 & 90 & 90 & 90 \\
\hline Volume $\left(\AA^{3}\right)$ & $2292.5(7)$ & $2387.7(8)$ & $2531.4(8)$ & $3217.1(10)$ \\
\hline Z & 4 & 4 & 4 & 4 \\
\hline Crystal size (mm) & $0.30 \times 0.30 \times 0.20$ & $0.40 \times 0.20 \times 0.20$ & $0.22 \times 0.14 \times 0.12$ & $0.30 \times 0.13 \times 0.11$ \\
\hline $\begin{array}{l}\text { Theta range for data } \\
\text { collection }\left({ }^{\circ}\right)\end{array}$ & 2.037 to 30.575 & 3.532 to 62.092 & 1.789 to 20.539 & 1.798 to 21.384 \\
\hline Reflections collected & 27495 & 18529 & 60407 & 92646 \\
\hline Independent reflections & 3684 & 3761 & 5185 & 7397 \\
\hline $\mathrm{R}_{\text {int }}$ & 0.0540 & 0.0430 & 0.0698 & 0.0607 \\
\hline $\begin{array}{l}\text { Data / restraints / } \\
\text { parameters }\end{array}$ & 3684 / 0 / 148 & $3761 / 0 / 260$ & 5185 / 0 / 289 & 7397 / 0 / 363 \\
\hline $\mathrm{R} 1[\mathrm{I}>2 \sigma(\mathrm{I})]$ & 0.0327 & 0.0267 & 0.0327 & 0.0275 \\
\hline wR2 (all data) & 0.0856 & 0.0705 & 0.0764 & 0.0632 \\
\hline$\Delta \rho_{\text {fin }}, e \AA^{-3}$ & $0.479 /-0.389$ & $0.284 /-0.234$ & $0.322 /-0.209$ & $0.393 /-0.329$ \\
\hline
\end{tabular}




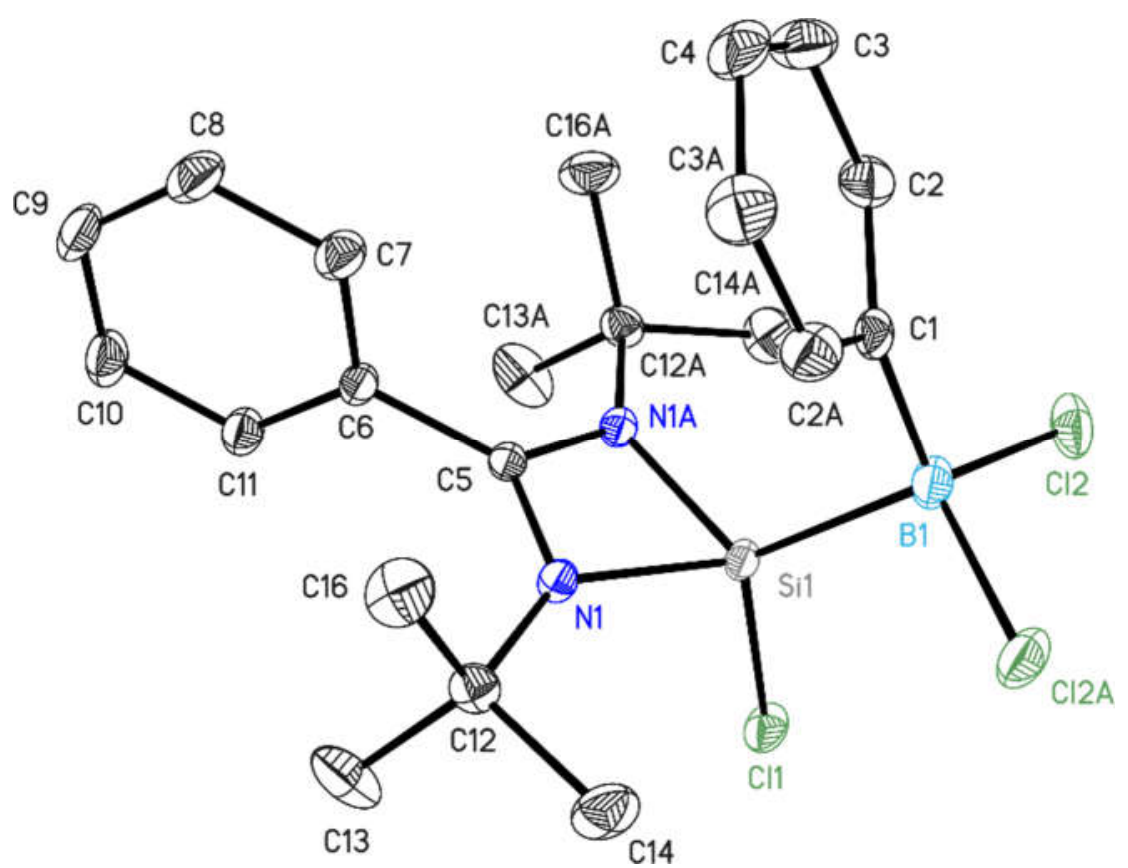

Figure S28 The structure of 1 with anisotropic displacement parameters at 50\% probability level. The hydrogen atoms are omitted for clarity.

Table S2. Bond lengths $[\AA]]$ and angles $\left[{ }^{\circ}\right]$ for 1 .

\begin{tabular}{|c|c|c|c|}
\hline $\mathrm{Cl}(1)-\mathrm{Si}(1)$ & $2.0464(6)$ & $C(10)-C(11)$ & $1.386(2)$ \\
\hline $\mathrm{Si}(1)-\mathrm{N}(1)$ & $1.8054(10)$ & & \\
\hline $\mathrm{Si}(1)-\mathrm{N}(1) \# 1$ & $1.8054(10)$ & $N(1)-S i(1)-N(1) \# 1$ & $72.80(6)$ \\
\hline $\mathrm{Si}(1)-\mathrm{B}(1)$ & $2.0369(19)$ & $N(1)-S i(1)-B(1)$ & $118.94(5)$ \\
\hline $\mathrm{Si}(1)-\mathrm{C}(5)$ & $2.2541(16)$ & $\mathrm{N}(1) \# 1-\mathrm{Si}(1)-\mathrm{B}(1)$ & $118.94(5)$ \\
\hline$C(1)-C(2) \# 1$ & $1.3969(15)$ & $\mathrm{N}(1)-\mathrm{Si}(1)-\mathrm{Cl}(1)$ & $108.72(3)$ \\
\hline$C(1)-C(2)$ & $1.3969(15)$ & $\mathrm{N}(1) \# 1-\mathrm{Si}(1)-\mathrm{Cl}(1)$ & $108.71(3)$ \\
\hline $\mathrm{C}(1)-\mathrm{B}(1)$ & $1.604(2)$ & $\mathrm{B}(1)-\mathrm{Si}(1)-\mathrm{Cl}(1)$ & $119.55(6)$ \\
\hline $\mathrm{B}(1)-\mathrm{Cl}(2)$ & $1.8749(12)$ & $N(1)-S i(1)-C(5)$ & $36.48(3)$ \\
\hline $\mathrm{B}(1)-\mathrm{Cl}(2) \# 1$ & $1.8749(12)$ & $N(1) \# 1-S i(1)-C(5)$ & $36.47(3)$ \\
\hline$N(1)-C(5)$ & $1.3400(13)$ & $B(1)-S i(1)-C(5)$ & $124.48(7)$ \\
\hline$N(1)-C(12)$ & $1.4853(15)$ & $\mathrm{Cl}(1)-\mathrm{Si}(1)-\mathrm{C}(5)$ & $115.98(4)$ \\
\hline$C(2)-C(3)$ & $1.3925(18)$ & $C(2) \# 1-C(1)-C(2)$ & $116.93(15)$ \\
\hline$C(3)-C(4)$ & $1.3862(18)$ & $\mathrm{C}(2) \# 1-\mathrm{C}(1)-\mathrm{B}(1)$ & $121.44(7)$ \\
\hline$C(5)-C(6)$ & $1.485(2)$ & $C(2)-C(1)-B(1)$ & $121.44(7)$ \\
\hline$C(6)-C(11)$ & $1.388(2)$ & $\mathrm{C}(1)-\mathrm{B}(1)-\mathrm{Cl}(2)$ & $113.10(7)$ \\
\hline$C(6)-C(7)$ & $1.397(2)$ & $\mathrm{C}(1)-\mathrm{B}(1)-\mathrm{Cl}(2) \# 1$ & $113.10(7)$ \\
\hline$C(12)-C(13)$ & $1.5243(17)$ & $\mathrm{Cl}(2)-\mathrm{B}(1)-\mathrm{Cl}(2) \# 1$ & $108.58(10)$ \\
\hline$C(12)-C(14)$ & $1.5257(18)$ & $C(1)-B(1)-S i(1)$ & $106.07(11)$ \\
\hline$C(12)-C(16)$ & $1.5261(17)$ & $\mathrm{Cl}(2)-\mathrm{B}(1)-\mathrm{Si}(1)$ & $107.86(6)$ \\
\hline$C(9)-C(10)$ & $1.383(3)$ & $\mathrm{Cl}(2) \# 1-\mathrm{B}(1)-\mathrm{Si}(1)$ & $107.86(6)$ \\
\hline$C(9)-C(8)$ & $1.386(3)$ & $C(5)-N(1)-C(12)$ & $131.01(10)$ \\
\hline$C(8)-C(7)$ & $1.390(2)$ & $\mathrm{C}(5)-\mathrm{N}(1)-\mathrm{Si}(1)$ & $90.31(7)$ \\
\hline
\end{tabular}




$\begin{array}{llll}\mathrm{C}(12)-\mathrm{N}(1)-\mathrm{Si}(1) & 138.68(8) & \mathrm{C}(7)-\mathrm{C}(6)-\mathrm{C}(5) & 118.38(14) \\ \mathrm{C}(3)-\mathrm{C}(2)-\mathrm{C}(1) & 121.82(13) & \mathrm{N}(1)-\mathrm{C}(12)-\mathrm{C}(13) & 109.48(9) \\ \mathrm{C}(4)-\mathrm{C}(3)-\mathrm{C}(2) & 119.96(13) & \mathrm{N}(1)-\mathrm{C}(12)-\mathrm{C}(14) & 105.37(10) \\ \mathrm{C}(3) \# 1-\mathrm{C}(4)-\mathrm{C}(3) & 119.51(16) & \mathrm{C}(13)-\mathrm{C}(12)-\mathrm{C}(14) & 109.68(11) \\ \mathrm{N}(1) \# 1-\mathrm{C}(5)-\mathrm{N}(1) & \mathrm{N}(1)-\mathrm{C}(12)-\mathrm{C}(16) & 111.67(9) \\ \mathrm{N}(1) \# 1-\mathrm{C}(5)-\mathrm{C}(6) & \mathrm{C}(13)-\mathrm{C}(12)-\mathrm{C}(16) & 110.75(11) \\ \mathrm{N}(1)-\mathrm{C}(5)-\mathrm{C}(6) & \mathrm{C}(14)-\mathrm{C}(12)-\mathrm{C}(16) & 109.76(10) \\ \mathrm{N}(1) \# 1-\mathrm{C}(5)-\mathrm{Si}(1) & \mathrm{C}(10)-\mathrm{C}(9)-\mathrm{C}(8) & 120.30(16) \\ \mathrm{N}(1)-\mathrm{C}(5)-\mathrm{Si}(1) & 106.17(13) & \mathrm{C}(9)-\mathrm{C}(8)-\mathrm{C}(7) & 120.33(16) \\ \mathrm{C}(6)-\mathrm{C}(5)-\mathrm{Si}(1) & 126.88(7) & \mathrm{C}(8)-\mathrm{C}(7)-\mathrm{C}(6) & 118.99(16) \\ \mathrm{C}(11)-\mathrm{C}(6)-\mathrm{C}(7) & 53.22(7) & \mathrm{C}(9)-\mathrm{C}(10)-\mathrm{C}(11) & 120.07(17) \\ \mathrm{C}(11)-\mathrm{C}(6)-\mathrm{C}(5) & 53.22(7) & \mathrm{C}(10)-\mathrm{C}(11)-\mathrm{C}(6) & 119.69(15)\end{array}$

Symmetry transformations used to generate equivalent atoms:

$\# 1 \mathrm{x},-\mathrm{y}+1 / 2, \mathrm{z}$

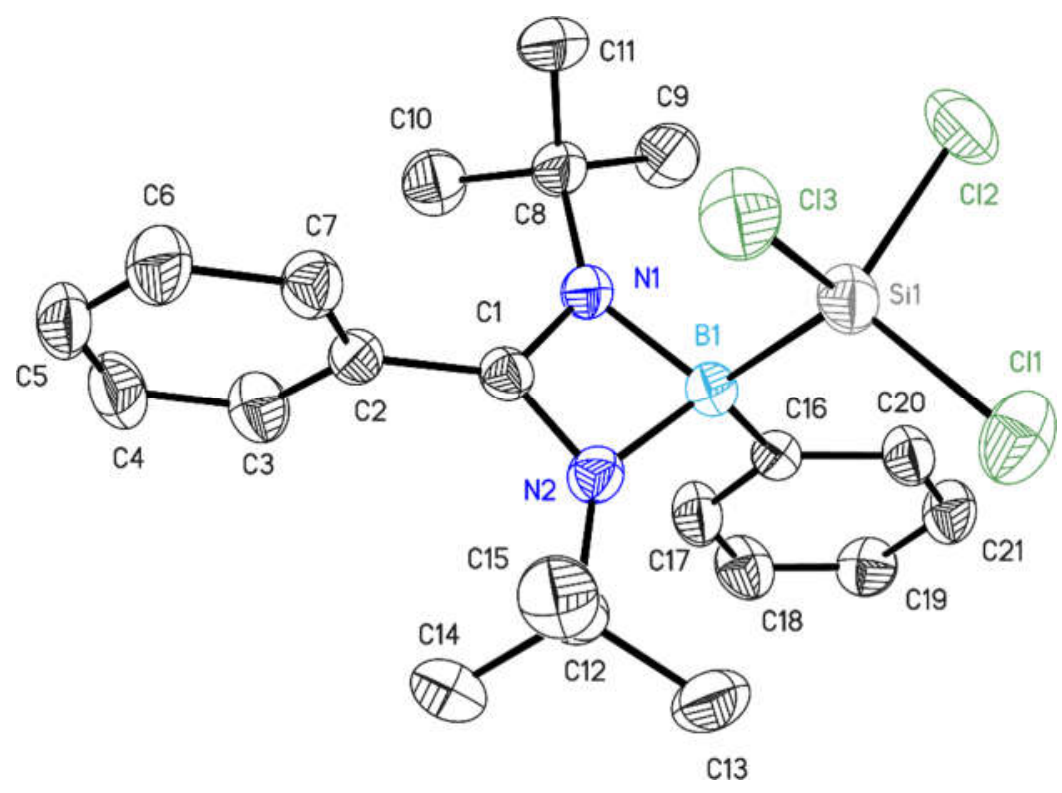

Figure S29. The asymmetric unit of 2 with anisotropic displacement parameters at $50 \%$ probability level. The hydrogen atoms are omitted for clarity. The structure was refined as an inversion twin with a fractional contribution of the minor domain of $0.374(14)^{10}$.

Table S3. Bond lengths $[\AA]$ and angles $\left[^{\circ}\right]$ for 2 .

$\begin{array}{llll}\mathrm{B}(1)-\mathrm{N}(1) & 1.584(3) & \mathrm{Si}(1)-\mathrm{Cl}(1) & 2.0823(11) \\ \mathrm{B}(1)-\mathrm{N}(2) & 1.586(3) & \mathrm{Si}(1)-\mathrm{Cl}(2) & 2.0859(10) \\ \mathrm{B}(1)-\mathrm{C}(1) & 1.597(3) & \mathrm{N}(1)-\mathrm{C}(1) & 1.338(3) \\ \mathrm{B}(1)-\mathrm{Si}(1) & 2.041(3) & \mathrm{N}(1)-\mathrm{C}(8) & 1.474(3) \\ \mathrm{B}(1)-\mathrm{C}(1) & 2.053(3) & \mathrm{N}(2)-\mathrm{C}(1) & 1.331(3) \\ \mathrm{Si}(1)-\mathrm{Cl}(3) & 2.0624(9) & \mathrm{N}(2)-\mathrm{C}(12) & 1.482(3)\end{array}$




\begin{tabular}{|c|c|c|c|}
\hline $\mathrm{C}(1)-\mathrm{C}(2)$ & $1.485(3)$ & $\mathrm{C}(8)-\mathrm{N}(1)-\mathrm{B}(1)$ & $139.47(19)$ \\
\hline$C(2)-C(7)$ & $1.385(4)$ & $\mathrm{C}(1)-\mathrm{N}(2)-\mathrm{C}(12)$ & $131.3(2)$ \\
\hline $\mathrm{C}(2)-\mathrm{C}(3)$ & $1.388(4)$ & $\mathrm{C}(1)-\mathrm{N}(2)-\mathrm{B}(1)$ & $89.04(18)$ \\
\hline$C(3)-C(4)$ & $1.385(4)$ & $\mathrm{C}(12)-\mathrm{N}(2)-\mathrm{B}(1)$ & $139.68(19)$ \\
\hline$C(4)-C(5)$ & $1.379(5)$ & $\mathrm{N}(2)-\mathrm{C}(1)-\mathrm{N}(1)$ & $100.93(19)$ \\
\hline$C(5)-C(6)$ & $1.370(5)$ & $\mathrm{N}(2)-\mathrm{C}(1)-\mathrm{C}(2)$ & $129.5(2)$ \\
\hline$C(6)-C(7)$ & $1.391(4)$ & $\mathrm{N}(1)-\mathrm{C}(1)-\mathrm{C}(2)$ & $129.5(2)$ \\
\hline $\mathrm{C}(8)-\mathrm{C}(11)$ & $1.526(4)$ & $\mathrm{N}(2)-\mathrm{C}(1)-\mathrm{B}(1)$ & $50.54(14)$ \\
\hline$C(8)-C(9)$ & $1.527(4)$ & $\mathrm{N}(1)-\mathrm{C}(1)-\mathrm{B}(1)$ & $50.46(13)$ \\
\hline $\mathrm{C}(8)-\mathrm{C}(10)$ & $1.532(4)$ & $\mathrm{C}(2)-\mathrm{C}(1)-\mathrm{B}(1)$ & 175.91(19) \\
\hline$C(12)-C(13)$ & $1.520(4)$ & $C(7)-C(2)-C(3)$ & $119.8(2)$ \\
\hline$C(12)-C(14)$ & $1.524(4)$ & $\mathrm{C}(7)-\mathrm{C}(2)-\mathrm{C}(1)$ & $119.2(2)$ \\
\hline$C(12)-C(15)$ & $1.530(4)$ & $\mathrm{C}(3)-\mathrm{C}(2)-\mathrm{C}(1)$ & $121.0(2)$ \\
\hline$C(16)-C(17)$ & $1.388(4)$ & $C(4)-C(3)-C(2)$ & $119.8(2)$ \\
\hline$C(16)-C(20)$ & $1.392(3)$ & $C(5)-C(4)-C(3)$ & $120.1(3)$ \\
\hline $\mathrm{C}(17)-\mathrm{C}(18)$ & $1.387(4)$ & $\mathrm{C}(6)-\mathrm{C}(5)-\mathrm{C}(4)$ & $120.4(2)$ \\
\hline$C(18)-C(19)$ & $1.377(4)$ & $C(5)-C(6)-C(7)$ & $120.0(3)$ \\
\hline$C(19)-C(21)$ & $1.379(4)$ & $C(2)-C(7)-C(6)$ & $119.9(2)$ \\
\hline \multirow[t]{2}{*}{$\mathrm{C}(20)-\mathrm{C}(21)$} & $1.391(4)$ & $\mathrm{N}(1)-\mathrm{C}(8)-\mathrm{C}(11)$ & $110.3(2)$ \\
\hline & & $\mathrm{N}(1)-\mathrm{C}(8)-\mathrm{C}(9)$ & $106.4(2)$ \\
\hline $\mathrm{N}(1)-\mathrm{B}(1)-\mathrm{N}(2)$ & $81.02(15)$ & $\mathrm{C}(11)-\mathrm{C}(8)-\mathrm{C}(9)$ & $110.7(2)$ \\
\hline $\mathrm{N}(1)-\mathrm{B}(1)-\mathrm{C}(16)$ & $118.12(19)$ & $\mathrm{N}(1)-\mathrm{C}(8)-\mathrm{C}(10)$ & $110.6(2)$ \\
\hline $\mathrm{N}(2)-\mathrm{B}(1)-\mathrm{C}(16)$ & $117.84(19)$ & $C(11)-C(8)-C(10)$ & $110.3(2)$ \\
\hline $\mathrm{N}(1)-\mathrm{B}(1)-\mathrm{Si}(1)$ & $109.09(15)$ & $\mathrm{C}(9)-\mathrm{C}(8)-\mathrm{C}(10)$ & $108.4(2)$ \\
\hline $\mathrm{N}(2)-\mathrm{B}(1)-\mathrm{Si}(1)$ & $108.00(15)$ & $\mathrm{N}(2)-\mathrm{C}(12)-\mathrm{C}(13)$ & $106.2(2)$ \\
\hline $\mathrm{C}(16)-\mathrm{B}(1)-\mathrm{Si}(1)$ & $117.17(16)$ & $\mathrm{N}(2)-\mathrm{C}(12)-\mathrm{C}(14)$ & $110.6(2)$ \\
\hline $\mathrm{N}(1)-\mathrm{B}(1)-\mathrm{C}(1)$ & $40.65(11)$ & $\mathrm{C}(13)-\mathrm{C}(12)-\mathrm{C}(14)$ & $109.1(2)$ \\
\hline $\mathrm{N}(2)-\mathrm{B}(1)-\mathrm{C}(1)$ & $40.41(11)$ & $\mathrm{N}(2)-\mathrm{C}(12)-\mathrm{C}(15)$ & $110.3(2)$ \\
\hline $\mathrm{C}(16)-\mathrm{B}(1)-\mathrm{C}(1)$ & $129.69(18)$ & $\mathrm{C}(13)-\mathrm{C}(12)-\mathrm{C}(15)$ & $110.6(2)$ \\
\hline $\mathrm{Si}(1)-\mathrm{B}(1)-\mathrm{C}(1)$ & $113.13(13)$ & $\mathrm{C}(14)-\mathrm{C}(12)-\mathrm{C}(15)$ & $110.1(2)$ \\
\hline $\mathrm{B}(1)-\mathrm{Si}(1)-\mathrm{Cl}(3)$ & $111.77(8)$ & $C(17)-C(16)-C(20)$ & $116.9(2)$ \\
\hline $\mathrm{B}(1)-\mathrm{Si}(1)-\mathrm{Cl}(1)$ & $115.43(8)$ & $\mathrm{C}(17)-\mathrm{C}(16)-\mathrm{B}(1)$ & $119.0(2)$ \\
\hline $\mathrm{Cl}(3)-\mathrm{Si}(1)-\mathrm{Cl}(1)$ & $104.59(4)$ & $\mathrm{C}(20)-\mathrm{C}(16)-\mathrm{B}(1)$ & $124.1(2)$ \\
\hline $\mathrm{B}(1)-\mathrm{Si}(1)-\mathrm{Cl}(2)$ & $116.55(8)$ & $C(18)-C(17)-C(16)$ & $122.0(2)$ \\
\hline $\mathrm{Cl}(3)-\mathrm{Si}(1)-\mathrm{Cl}(2)$ & $104.35(4)$ & $\mathrm{C}(19)-\mathrm{C}(18)-\mathrm{C}(17)$ & $120.2(3)$ \\
\hline $\mathrm{Cl}(1)-\mathrm{Si}(1)-\mathrm{Cl}(2)$ & $102.80(5)$ & $C(18)-C(19)-C(21)$ & 119.2(2) \\
\hline $\mathrm{C}(1)-\mathrm{N}(1)-\mathrm{C} 8)$ & $131.59(19)$ & $C(21)-C(20)-C(16)$ & $121.5(2)$ \\
\hline $\mathrm{C}(1)-\mathrm{N}(1)-\mathrm{B}(1)$ & $88.89(17)$ & $C(19)-C(21)-C(20)$ & $120.3(2)$ \\
\hline
\end{tabular}




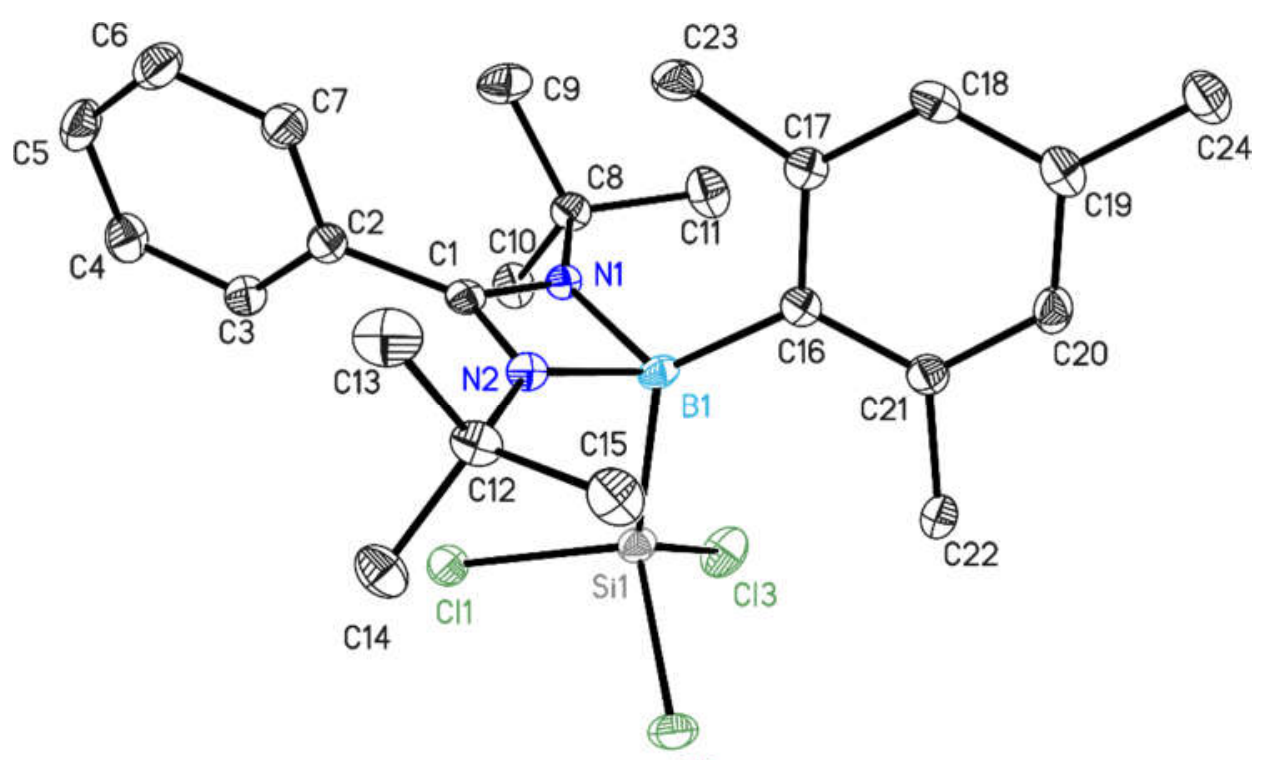

$\mathrm{Cl} 2$

Figure S30. The asymmetric unit of $\mathbf{3}$ with anisotropic displacement parameters at $50 \%$ probability level. The hydrogen atoms are omitted for clarity.

Table S4. Bond lengths $[\AA]]$ and angles $\left[{ }^{\circ}\right]$ for 3.

\begin{tabular}{|c|c|c|c|}
\hline $\mathrm{Cl}(1)-\mathrm{Si}(1)$ & $2.0775(7)$ & $C(12)-C(13)$ & $1.526(3)$ \\
\hline $\mathrm{Si}(1)-\mathrm{Cl}(2)$ & $2.0701(7)$ & $C(12)-C(14)$ & $1.531(2)$ \\
\hline $\mathrm{Si}(1)-\mathrm{Cl}(3)$ & $2.0703(7)$ & $C(16)-C(21)$ & $1.413(2)$ \\
\hline $\mathrm{Si}(1)-\mathrm{B}(1)$ & $2.076(2)$ & $C(16)-C(17)$ & $1.420(2)$ \\
\hline $\mathrm{C}(1)-\mathrm{N}(1)$ & $1.335(2)$ & $C(17)-C(18)$ & $1.392(2)$ \\
\hline $\mathrm{C}(1)-\mathrm{N}(2)$ & $1.338(2)$ & $C(17)-C(23)$ & $1.514(2)$ \\
\hline$C(1)-C(2)$ & $1.481(2)$ & $C(18)-C(19)$ & $1.382(3)$ \\
\hline$C(1)-B(1)$ & $2.068(3)$ & $C(19)-C(20)$ & $1.382(3)$ \\
\hline $\mathrm{B}(1)-\mathrm{N}(2)$ & $1.591(2)$ & $C(19)-C(24)$ & $1.505(2)$ \\
\hline $\mathrm{B}(1)-\mathrm{N}(1)$ & $1.600(2)$ & $C(20)-C(21)$ & $1.391(2)$ \\
\hline$B(1)-C(16)$ & $1.619(3)$ & $C(21)-C(22)$ & $1.510(3)$ \\
\hline $\mathrm{N}(1)-\mathrm{C}(8)$ & $1.487(2)$ & & \\
\hline$C(2)-C(3)$ & $1.390(2)$ & $\mathrm{Cl}(2)-\mathrm{Si}(1)-\mathrm{Cl}(3)$ & $104.16(3)$ \\
\hline$C(2)-C(7)$ & $1.394(2)$ & $\mathrm{Cl}(2)-\mathrm{Si}(1)-\mathrm{B}(1)$ & $120.57(6)$ \\
\hline$N(2)-C(12)$ & $1.486(2)$ & $\mathrm{Cl}(3)-\mathrm{Si}(1)-\mathrm{B}(1)$ & $117.67(6)$ \\
\hline$C(3)-C(4)$ & $1.384(2)$ & $\mathrm{Cl}(2)-\mathrm{Si}(1)-\mathrm{Cl}(1)$ & $100.44(3)$ \\
\hline$C(4)-C(5)$ & $1.384(3)$ & $\mathrm{Cl}(3)-\mathrm{Si}(1)-\mathrm{Cl}(1)$ & $101.87(3)$ \\
\hline$C(7)-C(6)$ & $1.384(3)$ & $\mathrm{B}(1)-\mathrm{Si}(1)-\mathrm{Cl}(1)$ & $109.30(6)$ \\
\hline$C(6)-C(5)$ & $1.384(3)$ & $N(1)-C(1)-N(2)$ & $100.92(14)$ \\
\hline$C(8)-C(11)$ & $1.524(3)$ & $N(1)-C(1)-C(2)$ & $127.46(15)$ \\
\hline$C(8)-C(10)$ & $1.527(2)$ & $N(2)-C(1)-C(2)$ & $131.60(16)$ \\
\hline$C(8)-C(9)$ & $1.529(2)$ & $N(1)-C(1)-B(1)$ & $50.69(10)$ \\
\hline$C(12)-C(15)$ & $1.523(2)$ & $N(2)-C(1)-B(1)$ & $50.30(10)$ \\
\hline
\end{tabular}




\begin{tabular}{|c|c|c|c|}
\hline$C(2)-C(1)-B(1)$ & $177.89(14)$ & $\mathrm{C}(17)-\mathrm{C}(16)-\mathrm{B}(1)$ & $120.26(15)$ \\
\hline$N(2)-B(1)-N(1)$ & $80.47(11)$ & $C(18)-C(17)-C(16)$ & 120.91(16) \\
\hline$N(2)-B(1)-C(16)$ & $119.25(14)$ & $C(18)-C(17)-C(23)$ & $115.09(16)$ \\
\hline$N(1)-B(1)-C(16)$ & $118.18(14)$ & $C(16)-C(17)-C(23)$ & $123.94(15)$ \\
\hline$N(2)-B(1)-C(1)$ & $40.32(8)$ & $C(19)-C(18)-C(17)$ & $122.77(17)$ \\
\hline$N(1)-B(1)-C(1)$ & $40.19(8)$ & $C(20)-C(19)-C(18)$ & $116.47(16)$ \\
\hline$C(16)-B(1)-C(1)$ & $130.43(14)$ & $C(20)-C(19)-C(24)$ & $121.78(17)$ \\
\hline$N(2)-B(1)-S i(1)$ & $102.33(11)$ & $C(18)-C(19)-C(24)$ & $121.75(17)$ \\
\hline$N(1)-B(1)-S i(1)$ & 99.66(11) & $C(19)-C(20)-C(21)$ & $122.80(17)$ \\
\hline$C(16)-B(1)-S i(1)$ & $126.46(13)$ & $C(20)-C(21)-C(16)$ & $121.16(16)$ \\
\hline $\mathrm{C}(1)-\mathrm{B}(1)-\mathrm{Si}(1)$ & $103.03(10)$ & $C(20)-C(21)-C(22)$ & $115.59(16)$ \\
\hline$C(1)-N(1)-C(8)$ & $132.30(14)$ & $C(16)-C(21)-C(22)$ & $123.22(16)$ \\
\hline$C(1)-N(1)-B(1)$ & $89.12(13)$ & & \\
\hline $\mathrm{C}(8)-\mathrm{N}(1)-\mathrm{B}(1)$ & $138.56(14)$ & $\mathrm{Cl}(2)-\mathrm{Si}(1)-\mathrm{Cl}(3)$ & 104.16(3) \\
\hline$C(3)-C(2)-C(7)$ & 119.34(16) & $\mathrm{Cl}(2)-\mathrm{Si}(1)-\mathrm{B}(1)$ & $120.57(6)$ \\
\hline$C(3)-C(2)-C(1)$ & $120.54(15)$ & $\mathrm{Cl}(3)-\mathrm{Si}(1)-\mathrm{B}(1)$ & $117.67(6)$ \\
\hline$C(7)-C(2)-C(1)$ & $119.86(15)$ & $\mathrm{Cl}(2)-\mathrm{Si}(1)-\mathrm{Cl}(1)$ & $100.44(3)$ \\
\hline $\mathrm{C}(1)-\mathrm{N}(2)-\mathrm{C}(12)$ & $133.24(15)$ & $\mathrm{Cl}(3)-\mathrm{Si}(1)-\mathrm{Cl}(1)$ & $101.87(3)$ \\
\hline$C(1)-N(2)-B(1)$ & $89.38(13)$ & $\mathrm{B}(1)-\mathrm{Si}(1)-\mathrm{Cl}(1)$ & $109.30(6)$ \\
\hline $\mathrm{C}(12)-\mathrm{N}(2)-\mathrm{B}(1)$ & $136.87(13)$ & $N(1)-C(1)-N(2)$ & $100.92(14)$ \\
\hline$C(4)-C(3)-C(2)$ & $120.08(17)$ & $N(1)-C(1)-C(2)$ & $127.46(15)$ \\
\hline$C(3)-C(4)-C(5)$ & $120.39(17)$ & $N(2)-C(1)-C(2)$ & $131.60(16)$ \\
\hline$C(6)-C(7)-C(2)$ & $120.26(17)$ & $N(1)-C(1)-B(1)$ & $50.69(10)$ \\
\hline$C(5)-C(6)-C(7)$ & $120.08(18)$ & $N(2)-C(1)-B(1)$ & $50.30(10)$ \\
\hline$C(4)-C(5)-C(6)$ & $119.84(17)$ & $C(2)-C(1)-B(1)$ & $177.89(14)$ \\
\hline$N(1)-C(8)-C(11)$ & $106.10(14)$ & $N(2)-B(1)-N(1)$ & $80.47(11)$ \\
\hline$N(1)-C(8)-C(10)$ & $111.07(14)$ & $N(2)-B(1)-C(16)$ & $119.25(14)$ \\
\hline$C(11)-C(8)-C(10)$ & $110.07(15)$ & $N(1)-B(1)-C(16)$ & $118.18(14)$ \\
\hline$N(1)-C(8)-C(9)$ & $111.83(14)$ & $N(2)-B(1)-C(1)$ & $40.32(8)$ \\
\hline$C(11)-C(8)-C(9)$ & $109.51(15)$ & $N(1)-B(1)-C(1)$ & $40.19(8)$ \\
\hline$C(10)-C(8)-C(9)$ & $108.25(15)$ & $C(16)-B(1)-C(1)$ & $130.43(14)$ \\
\hline$N(2)-C(12)-C(15)$ & $106.00(14)$ & $N(2)-B(1)-S i(1)$ & $102.33(11)$ \\
\hline$N(2)-C(12)-C(13)$ & $110.98(14)$ & $N(1)-B(1)-S i(1)$ & $99.66(11)$ \\
\hline$C(15)-C(12)-C(13)$ & $109.62(15)$ & $C(16)-B(1)-S i(1)$ & $126.46(13)$ \\
\hline$N(2)-C(12)-C(14)$ & $110.84(14)$ & $C(1)-B(1)-S i(1)$ & $103.03(10)$ \\
\hline$C(15)-C(12)-C(14)$ & $110.25(15)$ & $\mathrm{C}(1)-\mathrm{N}(1)-\mathrm{C}(8)$ & $132.30(14)$ \\
\hline$C(13)-C(12)-C(14)$ & $109.12(16)$ & $\mathrm{C}(1)-\mathrm{N}(1)-\mathrm{B}(1)$ & $89.12(13)$ \\
\hline$C(21)-C(16)-C(17)$ & $115.75(15)$ & $\mathrm{C}(8)-\mathrm{N}(1)-\mathrm{B}(1)$ & $138.56(14)$ \\
\hline $\mathrm{C}(21)-\mathrm{C}(16)-\mathrm{B}(1)$ & $123.99(15)$ & $C(3)-C(2)-C(7)$ & 119.34(16) \\
\hline
\end{tabular}




$\begin{array}{llll}\mathrm{C}(3)-\mathrm{C}(2)-\mathrm{C}(1) & 120.54(15) & \mathrm{C}(15)-\mathrm{C}(12)-\mathrm{C}(13) & 109.62(15) \\ \mathrm{C}(7)-\mathrm{C}(2)-\mathrm{C}(1) & 119.86(15) & \mathrm{N}(2)-\mathrm{C}(12)-\mathrm{C}(14) & 110.84(14) \\ \mathrm{C}(1)-\mathrm{N}(2)-\mathrm{C}(12) & 133.24(15) & \mathrm{C}(15)-\mathrm{C}(12)-\mathrm{C}(14) & 110.25(15) \\ \mathrm{C}(1)-\mathrm{N}(2)-\mathrm{B}(1) & 89.38(13) & \mathrm{C}(13)-\mathrm{C}(12)-\mathrm{C}(14) & 109.12(16) \\ \mathrm{C}(12)-\mathrm{N}(2)-\mathrm{B}(1) & \mathrm{C}(21)-\mathrm{C}(16)-\mathrm{C}(17) & 115.75(15) \\ \mathrm{C}(4)-\mathrm{C}(3)-\mathrm{C}(2) & 136.87(13) & \mathrm{C}(21)-\mathrm{C}(16)-\mathrm{B}(1) & 123.99(15) \\ \mathrm{C}(3)-\mathrm{C}(4)-\mathrm{C}(5) & \mathrm{C}(17)-\mathrm{C}(16)-\mathrm{B}(1) & 120.26(15) \\ \mathrm{C}(6)-\mathrm{C}(7)-\mathrm{C}(2) & 120.08(17) & \mathrm{C}(18)-\mathrm{C}(17)-\mathrm{C}(16) & 120.91(16) \\ \mathrm{C}(5)-\mathrm{C}(6)-\mathrm{C}(7) & \mathrm{C}(18)-\mathrm{C}(17)-\mathrm{C}(23) & 115.09(16) \\ \mathrm{C}(4)-\mathrm{C}(5)-\mathrm{C}(6) & 120.39(17) & \mathrm{C}(16)-\mathrm{C}(17)-\mathrm{C}(23) & 123.94(15) \\ \mathrm{N}(1)-\mathrm{C}(8)-\mathrm{C}(11) & 120.26(17) & \mathrm{C}(19)-\mathrm{C}(18)-\mathrm{C}(17) & 122.77(17) \\ \mathrm{N}(1)-\mathrm{C}(8)-\mathrm{C}(10) & 120.08(18) & \mathrm{C}(20)-\mathrm{C}(19)-\mathrm{C}(18) & 116.47(16) \\ \mathrm{C}(11)-\mathrm{C}(8)-\mathrm{C}(10) & 119.84(17) & \mathrm{C}(20)-\mathrm{C}(19)-\mathrm{C}(24) & 121.78(17) \\ \mathrm{N}(1)-\mathrm{C}(8)-\mathrm{C}(9) & 106.10(14) & \mathrm{C}(18)-\mathrm{C}(19)-\mathrm{C}(24) & 121.75(17) \\ \mathrm{C}(11)-\mathrm{C}(8)-\mathrm{C}(9) & 111.07(14) & \mathrm{C}(19)-\mathrm{C}(20)-\mathrm{C}(21) & 122.80(17) \\ \mathrm{C}(10)-\mathrm{C}(8)-\mathrm{C}(9) & 110.07(15) & \mathrm{C}(20)-\mathrm{C}(21)-\mathrm{C}(16) & 121.16(16) \\ \mathrm{N}(2)-\mathrm{C}(12)-\mathrm{C}(15) & 111.83(14) & \mathrm{C}(20)-\mathrm{C}(21)-\mathrm{C}(22) & 115.59(16) \\ \mathrm{N}(2)-\mathrm{C}(12)-\mathrm{C}(13) & \mathrm{C}(16)-\mathrm{C}(21)-\mathrm{C}(22) & 123.22(16)\end{array}$

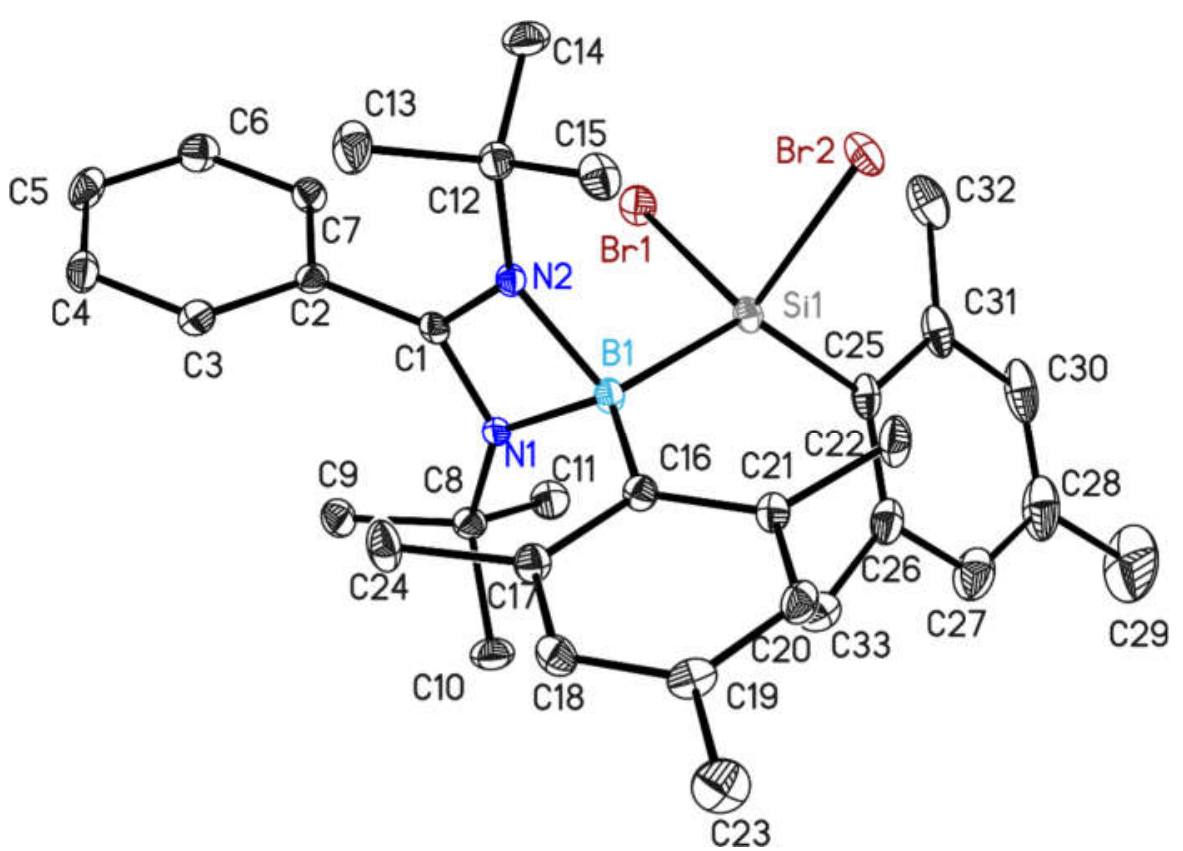

Figure S31. The asymmetric unit of 7 with anisotropic displacement parameters at $50 \%$ probability level. The hydrogen atoms are omitted for clarity.

Table S5. Bond lengths $[\AA]$ and angles $\left[^{\circ}\right]$ for 7 .

$\begin{array}{lllr}\mathrm{Br}(1)-\mathrm{Si}(1) & 2.2738(8) & \mathrm{N}(1)-\mathrm{C}(1) & 1.339(2) \\ \mathrm{Si}(1)-\mathrm{C}(25) & 1.919(2) & \mathrm{N}(1)-\mathrm{C}(8) & 1.488(2) \\ \mathrm{Si}(1)-\mathrm{B}(1) & 2.139(2) & \mathrm{N}(1)-\mathrm{B}(1) & 1.625(3) \\ \mathrm{Si}(1)-\mathrm{Br}(2) & 2.2784(7) & \mathrm{C}(1)-\mathrm{N}(2) & 1.333(2)\end{array}$




\begin{tabular}{|c|c|c|c|}
\hline$C(1)-C(2)$ & $1.487(3)$ & $\mathrm{B}(1)-\mathrm{Si}(1)-\mathrm{Br}(2)$ & $112.28(6)$ \\
\hline $\mathrm{C}(1)-\mathrm{B}(1)$ & $2.070(3)$ & $\operatorname{Br}(1)-\mathrm{Si}(1)-\operatorname{Br}(2)$ & $99.41(3)$ \\
\hline $\mathrm{B}(1)-\mathrm{N}(2)$ & $1.592(3)$ & $C(1)-N(1)-C(8)$ & $132.08(16)$ \\
\hline$B(1)-C(16)$ & $1.633(3)$ & $C(1)-N(1)-B(1)$ & $88.09(14)$ \\
\hline$N(2)-C(12)$ & $1.489(2)$ & $\mathrm{C}(8)-\mathrm{N}(1)-\mathrm{B}(1)$ & $139.72(15)$ \\
\hline$C(2)-C(7)$ & $1.391(3)$ & $N(2)-C(1)-N(1)$ & $101.87(16)$ \\
\hline$C(2)-C(3)$ & $1.393(3)$ & $N(2)-C(1)-C(2)$ & $127.98(17)$ \\
\hline$C(3)-C(4)$ & $1.386(3)$ & $N(1)-C(1)-C(2)$ & $130.08(17)$ \\
\hline$C(4)-C(5)$ & $1.380(3)$ & $N(2)-C(1)-B(1)$ & $50.25(11)$ \\
\hline$C(5)-C(6)$ & $1.386(3)$ & $\mathrm{N}(1)-\mathrm{C}(1)-\mathrm{B}(1)$ & $51.66(11)$ \\
\hline$C(6)-C(7)$ & $1.390(3)$ & $C(2)-C(1)-B(1)$ & $178.17(16)$ \\
\hline$C(9)-C(8)$ & $1.536(3)$ & $\mathrm{N}(2)-\mathrm{B}(1)-\mathrm{N}(1)$ & $80.31(13)$ \\
\hline$C(8)-C(11)$ & $1.530(3)$ & $N(2)-B(1)-C(16)$ & $114.41(15)$ \\
\hline$C(8)-C(10)$ & $1.531(3)$ & $N(1)-B(1)-C(16)$ & $119.03(16)$ \\
\hline$C(12)-C(14)$ & $1.526(3)$ & $N(2)-B(1)-C(1)$ & $40.09(9)$ \\
\hline$C(12)-C(13)$ & $1.531(3)$ & $N(1)-B(1)-C(1)$ & $40.25(9)$ \\
\hline$C(12)-C(15)$ & $1.533(3)$ & $C(16)-B(1)-C(1)$ & $127.18(15)$ \\
\hline$C(16)-C(21)$ & $1.424(3)$ & $N(2)-B(1)-S i(1)$ & $104.92(12)$ \\
\hline$C(16)-C(17)$ & $1.425(3)$ & $N(1)-B(1)-S i(1)$ & $104.14(12)$ \\
\hline$C(17)-C(18)$ & $1.390(3)$ & $C(16)-B(1)-S i(1)$ & $124.75(14)$ \\
\hline$C(17)-C(24)$ & $1.515(3)$ & $C(1)-B(1)-S i(1)$ & $107.97(11)$ \\
\hline$C(18)-C(19)$ & $1.386(3)$ & $C(1)-N(2)-C(12)$ & $131.47(16)$ \\
\hline$C(19)-C(20)$ & $1.380(3)$ & $\mathrm{C}(1)-\mathrm{N}(2)-\mathrm{B}(1)$ & $89.66(14)$ \\
\hline$C(19)-C(23)$ & $1.504(3)$ & $C(12)-N(2)-B(1)$ & $138.82(15)$ \\
\hline$C(20)-C(21)$ & $1.396(3)$ & $C(7)-C(2)-C(3)$ & $119.15(18)$ \\
\hline$C(21)-C(22)$ & $1.503(3)$ & $C(7)-C(2)-C(1)$ & $121.33(17)$ \\
\hline$C(25)-C(26)$ & $1.412(3)$ & $C(3)-C(2)-C(1)$ & $119.52(17)$ \\
\hline$C(25)-C(31)$ & $1.430(3)$ & $C(4)-C(3)-C(2)$ & $120.60(19)$ \\
\hline$C(26)-C(27)$ & $1.399(3)$ & $C(5)-C(4)-C(3)$ & $119.98(19)$ \\
\hline$C(26)-C(33)$ & $1.510(3)$ & $C(4)-C(5)-C(6)$ & $119.98(19)$ \\
\hline$C(27)-C(28)$ & $1.375(4)$ & $C(5)-C(6)-C(7)$ & $120.28(19)$ \\
\hline$C(28)-C(30)$ & $1.381(4)$ & $C(6)-C(7)-C(2)$ & $120.00(19)$ \\
\hline$C(28)-C(29)$ & $1.513(3)$ & $N(1)-C(8)-C(11)$ & $110.13(15)$ \\
\hline$C(30)-C(31)$ & $1.396(3)$ & $\mathrm{N}(1)-\mathrm{C}(8)-\mathrm{C}(10)$ & $108.30(15)$ \\
\hline$C(31)-C(32)$ & $1.508(3)$ & $C(11)-C(8)-C(10)$ & $110.07(17)$ \\
\hline$C(25)-S i(1)-B(1)$ & $128.82(9)$ & $N(1)-C(8)-C(9)$ & $112.92(16)$ \\
\hline $\mathrm{C}(25)-\mathrm{Si}(1)-\mathrm{Br}(1)$ & $105.25(6)$ & $C(11)-C(8)-C(9)$ & $107.76(16)$ \\
\hline$B(1)-S i(1)-B r(1)$ & $104.43(6)$ & $\mathrm{C}(10)-\mathrm{C}(8)-\mathrm{C}(9)$ & $107.62(16)$ \\
\hline$C(25)-S i(1)-B r(2)$ & $102.77(7)$ & $N(2)-C(12)-C(14)$ & $109.94(15)$ \\
\hline
\end{tabular}




\begin{tabular}{|c|c|c|c|}
\hline $\mathrm{N}(2)-\mathrm{C}(12)-\mathrm{C}(13)$ & $112.18(16)$ & $C(20)-C(21)-C(22)$ & $114.14(17)$ \\
\hline$C(14)-C(12)-C(13)$ & $108.78(18)$ & $C(16)-C(21)-C(22)$ & $124.48(17)$ \\
\hline$N(2)-C(12)-C(15)$ & $106.47(16)$ & $C(26)-C(25)-C(31)$ & 116.93(19) \\
\hline$C(14)-C(12)-C(15)$ & $111.02(17)$ & $\mathrm{C}(26)-\mathrm{C}(25)-\mathrm{Si}(1)$ & $121.47(15)$ \\
\hline$C(13)-C(12)-C(15)$ & $108.45(17)$ & $\mathrm{C}(31)-\mathrm{C}(25)-\mathrm{Si}(1)$ & $121.52(16)$ \\
\hline$C(21)-C(16)-C(17)$ & $115.34(17)$ & $\mathrm{C}(27)-\mathrm{C}(26)-\mathrm{C}(25)$ & $121.0(2)$ \\
\hline$C(21)-C(16)-B(1)$ & $123.76(17)$ & $C(27)-C(26)-C(33)$ & $114.5(2)$ \\
\hline$C(17)-C(16)-B(1)$ & $120.74(16)$ & $\mathrm{C}(25)-\mathrm{C}(26)-\mathrm{C}(33)$ & $124.48(18)$ \\
\hline$C(18)-C(17)-C(16)$ & $121.13(18)$ & $\mathrm{C}(28)-\mathrm{C}(27)-\mathrm{C}(26)$ & $122.1(2)$ \\
\hline$C(18)-C(17)-C(24)$ & $113.82(17)$ & $C(27)-C(28)-C(30)$ & $117.2(2)$ \\
\hline$C(16)-C(17)-C(24)$ & $124.96(17)$ & $C(27)-C(28)-C(29)$ & $121.7(3)$ \\
\hline$C(19)-C(18)-C(17)$ & $122.84(18)$ & $C(30)-C(28)-C(29)$ & $121.1(2)$ \\
\hline$C(20)-C(19)-C(18)$ & $116.76(18)$ & $\mathrm{C}(28)-\mathrm{C}(30)-\mathrm{C}(31)$ & $123.5(2)$ \\
\hline$C(20)-C(19)-C(23)$ & 121.92(19) & $C(30)-C(31)-C(25)$ & 119.2(2) \\
\hline$C(18)-C(19)-C(23)$ & $121.30(19)$ & $C(30)-C(31)-C(32)$ & $115.2(2)$ \\
\hline$C(19)-C(20)-C(21)$ & $122.56(18)$ & $C(25)-C(31)-C(32)$ & $125.61(19)$ \\
\hline$C(20)-C(21)-C(16)$ & $121.31(18)$ & & \\
\hline
\end{tabular}




\section{References}

1 Sen, S. S.; Roesky, H. W.; Stern, D.; Henn, J.; Stalke, D. High Yield Access to Silylene RSiCl $\left(\mathrm{R}=\mathrm{PhC}(\mathrm{N} t \mathrm{Bu})_{2}\right)$ and Its Reactivity toward Alkyne: Synthesis of Stable Disilacyclobutene. J. Am. Chem. Soc. 2010, 132, 1123-1126.

2 Cabeza, J. A.; García-Álvarez, P.; González-Álvarez, L. Facile Cyclometallation of a Mesitylsilylene: Synthesis and Preliminary Catalytic Activity of Iridium(III) and Iridium(V) Iridasilacyclopentenes. Chem. Commun. 2017, 53, 10275-10278.

3 Li, B.-L.; Goodman, M. A.; Neilson, R. H. Synthesis and Stereochemistry of Some Alkyl[bis(trimethylsilyl)amino]boranes. Inorg Chem. 1984, 23, 1368-1371.

4 Sundararaman, A.; Jäkle, F. A Comparative Study of Base-free Arylcopper Reagents for the Transfer of Aryl Groups to Boron Halides. J. Organomet. Chem. 2003, 681, 134-142.

5 Bruker AXS Inc., in Bruker Apex CCD, SAINT v8.30C (Ed.: Bruker AXS Inst. Inc.), WI, USA, Madison, 2013.

6 Krause, L.; Herbst-Irmer, R.; Sheldrick, G. M.; Stalke, D. Comparison of Silver and Molybdenum Microfocus X-Ray Sources for Single-Crystal Structure Determination. J. Appl. Crystallogr. 2015, 48, 3-10.

7 Sheldrick, G. M. SHELXT-Integrated Space-Group and Crystal-Structure Determination. Acta Crystallogr. 2015, A71, 3-8.

8 Sheldrick, G. M. Crystal Structure Refinement with SHELXL. Acta Crystallogr. 2015, C71, 3-8.

9 Hübschle, C. B.; Sheldrick, G. M.; Dittrich, B. ShelXle: A Qt Graphical User Interface for SHELXL. J. Appl. Crystallogr. 2011, 44, 1281-1284.

10 Parsons, S.; Flack, H. D.; Wagner, T. Use of Intensity Quotients and Differences in Absolute Structure Refinement. Acta Crystallogr. 2013, B69, 249-259. 